\title{
Diversity of short interspersed nuclear elements (SINEs) in lepidopteran insects and evidence of horizontal SINE transfer between baculovirus and lepidopteran hosts
}

Guangjie Han ${ }^{1,2}$, Nan Zhang ${ }^{1}$, Heng Jiang ${ }^{1}$, Xiangkun Meng ${ }^{1}$, Kun Qian ${ }^{1}$, Yang Zheng ${ }^{1}$, Jian Xu ${ }^{2 *}$ and Jianjun Wang ${ }^{1,3^{*}}$ (D)

\begin{abstract}
Background: Short interspersed nuclear elements (SINEs) belong to non-long terminal repeat (non-LTR) retrotransposons, which can mobilize dependent on the help of counterpart long interspersed nuclear elements (LINEs). Although 234 SINEs have been identified so far, only 23 are from insect species (SINEbase: http://sines.eimb. $\mathrm{ru} /$ ).

Results: Here, five SINEs were identified from the genome of Plutella xylostella, among which PXSE1, PXSE2 and PXSE3 were tRNA-derived SINES, PXSE4 and PXSE5 were 5S RNA-derived SINEs. A total of 18 related SINEs were further identified in 13 lepidopteran insects and a baculovirus. The 3'-tail of PXSE5 shares highly identity with that of LINE retrotransposon, PXLINE1. The analysis of relative age distribution profiles revealed that PXSE1 is a relatively young retrotransposon in the genome of $P$. xylostella and was generated by recent explosive amplification. Integration pattern analysis showed that SINEs in P. xylostella prefer to insert into or accumulate in introns and regions $5 \mathrm{~kb}$ downstream of genes. In particular, the PXSE7-like element, SINPVSE1, in Spodoptera litura nucleopolyhedrovirus II genome is highly identical to SFSE1 in Spodoptera frugiperda, SlittSE1 in Spodoptera littoralis, and SlituSE1 in Spodoptera litura, suggesting the occurrence of horizontal transfer.
\end{abstract}

Conclusions: Lepidopteran insect genomes harbor a diversity of SINEs. The retrotransposition activity and copy number of these SINEs varies considerably between host lineages and SINE lineages. Host-parasite interactions facilitate the horizontal transfer of SINE between baculovirus and its lepidopteran hosts.

Keywords: Short interspersed nuclear element (SINE), Horizontal transfer, Plutella xylostella, Retrotransposon, Long interspersed nuclear elements (LINEs)

\footnotetext{
*Correspondence: bio-xj@163.com; wangij@yzu.edu.cn

${ }^{2}$ Jiangsu Lixiahe District Institute of Agricultural Sciences, Yangzhou 225008,

China

${ }^{1}$ College of Horticulture and Plant Protection, Yangzhou University, Yangzhou 225009, China

Full list of author information is available at the end of the article
}

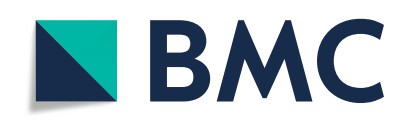

(- The Author(s). 2021 Open Access This article is licensed under a Creative Commons Attribution 4.0 International License, which permits use, sharing, adaptation, distribution and reproduction in any medium or format, as long as you give appropriate credit to the original author(s) and the source, provide a link to the Creative Commons licence, and indicate if changes were made. The images or other third party material in this article are included in the article's Creative Commons licence, unless indicated otherwise in a credit line to the material. If material is not included in the article's Creative Commons licence and your intended use is not permitted by statutory regulation or exceeds the permitted use, you will need to obtain permission directly from the copyright holder. To view a copy of this licence, visit http://creativecommons.org/licenses/by/4.0/ The Creative Commons Public Domain Dedication waiver (http://creativecommons.org/publicdomain/zero/1.0/) applies to the data made available in this article, unless otherwise stated in a credit line to the data. 


\section{Background}

Short interspersed nuclear elements (SINEs) are Class I transposable elements (TEs) that propagate by a copyand-paste mechanism $[1,2]$. SINEs are evolutionarily derived from endogenous RNA polymerase III (Pol III) transcripts [3]. While mammalian SINEs, such as B1 and Alu, are originated from 7SL RNAs, other eukaryotes primarily harbor tRNA-like SINEs [4], and SINEs originated from $5 \mathrm{~S}$ rRNA have been found in zebrafish, fruit bats, and springhare $[5,6]$. Recently, SINEs derived from small nuclear RNA (snRNA) (SINEU) and the 3 '-end of the large ribosomal subunit (LSU or $28 \mathrm{~S}$ rDNA) (SINE28) have been identified in avian, crocodilian and mammalian genomes, respectively [7-9]. The characteristic features of SINEs include a $5^{\prime}$ terminal RNArelated region which contains an internal Pol III promoter, a central region, and a 3 '-tail that is of variable length and recognized by the reverse transcriptase (RT) of autonomous partner long nuclear interspersed element (LINE) during retrotransposition [3]. The SINEs promoters originated from tRNA and 7SL RNA comprise box A and B motif, whereas $5 \mathrm{~S}$ rRNA-derived SINE promoters have three boxes such as A, IE and C [10].

As non-autonomous retrotransposon, the replication rate and survival of a SINE is dependent on the partner LINE activity, and its genomic copy number varies greatly between families and host species. For example, as high as 1 million copies of $A l u$ elements have been identified in the human genome [11], whereas only two copies of ZmSINE3 were detected in Zea mays [12]. On the other hand, the number of SINE families within a genome is also highly variable, ranging from a single SINE family in the Vitaceae to 22 SINE families in the Amaranthaceae [13]. Interestingly, unlike LINEs, the distribution of a SINE family is generally restricted to a certain taxonomic group such as orders/families [3, 4, 14], suggesting that SINEs are one of the major genetic elements that determine a clade-specific genomic composition.

Transposable elements play an important role in the epigenetic regulation of the genome and generation of genomic novelty. A growing body of evidence has recently accumulated indicating that SINEs have a deep impact on genome organization and gene structure by generating regulatory elements for gene expression $[15$, 16], exon skipping and alternative splicing [17], alternative polyadenylation signals $[18,19]$, and even functional RNA genes [20, 21]. For example, an Alu SINE inserted into human pluripotency-associated transcript 5 (HPAT5) regulated related microRNAs through its let-7 binding site, which is essential for inner cell mass formation during early embryonic development [22].

While SINEs have been well characterized in human [23], other mammals [24] and plants [25], and currently about 200 SINE families/subfamilies are identified in various clades in Metazoa, as reported in Repbase [26] and in SINEBase [2], information on insect SINEs is still limited [27-33]. Recent improvements in both genome sequencing and assembly methodologies have led to increasing high-quality insect genome assemblies, which provides the opportunity to identify novel SINEs. However, due to their minimal sequence feature, the lack of coding capacity, and high sequence heterogeneity, annotations of SINE are often incomplete or missing. Here, we described three tRNA-derived SINE families and two 5S rRNA-derived SINE families in the diamondback moth (DBM), Plutella xylostella (L.), which is one of the most damaging insect pests of cruciferous vegetables around the world. We investigated the structures and insertion regions of these SINEs. The distribution of these SINEs in other lepidopteran insect species was also surveyed.

\section{Results \\ Novel tRNA-derived SINE retrotransposons, PXSE1, PXSE2 and PXSE3, in P. xylostella}

A novel tRNA-derived SINE, PxSE1, was identified by homology search in DBM genome database (Additional file 1: Figure S1). A total of 68 full length copies homologous to PxSE1 were used to reconstruct the consensus sequence of PXSE1 (Accession numbers: MW068006-MW068073). The PXSE1 is 263 bp long, includes GT dinucleotide repeats at 3 '-tail and a 72-bp tRNA-related region at the $5^{\prime}$-end with $64 \%$ identity to 72-bp tRNA ${ }^{\text {Arg }}$ of Drosophila melanogaster, which contains box A and box B of the RNA Pol III promoter (Figs. 1 and 2a, Additional file 2: Figure S2). The boundary of PXSE1 was further defined by the alignment of a PXSE1 element and its empty site sequence (Additional file 3: Figure S3). Using the PxSE1 as the query, a total of 6208 copies were identified in DBM genome (Table 1). The average divergence is 0.035 in all PXSE1 copies (Table 1), indicating a recent invasion time.

Using PxSE1 as query, two additional tRNA-derived SINEs, PxSE2 and PxSE3, were identified by database searches. The consensus sequences of PxSE2 and PxSE3 were reconstructed using the same methods as described above (Accession numbers: MW068074-MW068156, Additional file 2: Figure S2 and Additional file 3: Figure S3). The PxSE2 is 263 bp long, includes a 143 bp 3 '-end sequence, which is different from PxSE1, but has $67.5 \%$ identity with $B m S E$. The 72-bp tRNA-related region of PXSE2 is $93.4 \%$ identical to PxSE1 (Figs. 1 and 2a). Interestingly, PxSE2 has a $44 \mathrm{bp}$ conserved central domain with $93.2 \%$ identity to PxSE1 (Fig. 2a). The PxSE3 is 339 bp long, includes a 72-bp tRNA-related region with $66.5 \%$ identity to tRNA ${ }^{\text {Arg }}$ of $D$. melanogaster, and has $79.3 \%$ identity with the $222 \mathrm{bp}$ sequence at $5^{\prime}$-end of 


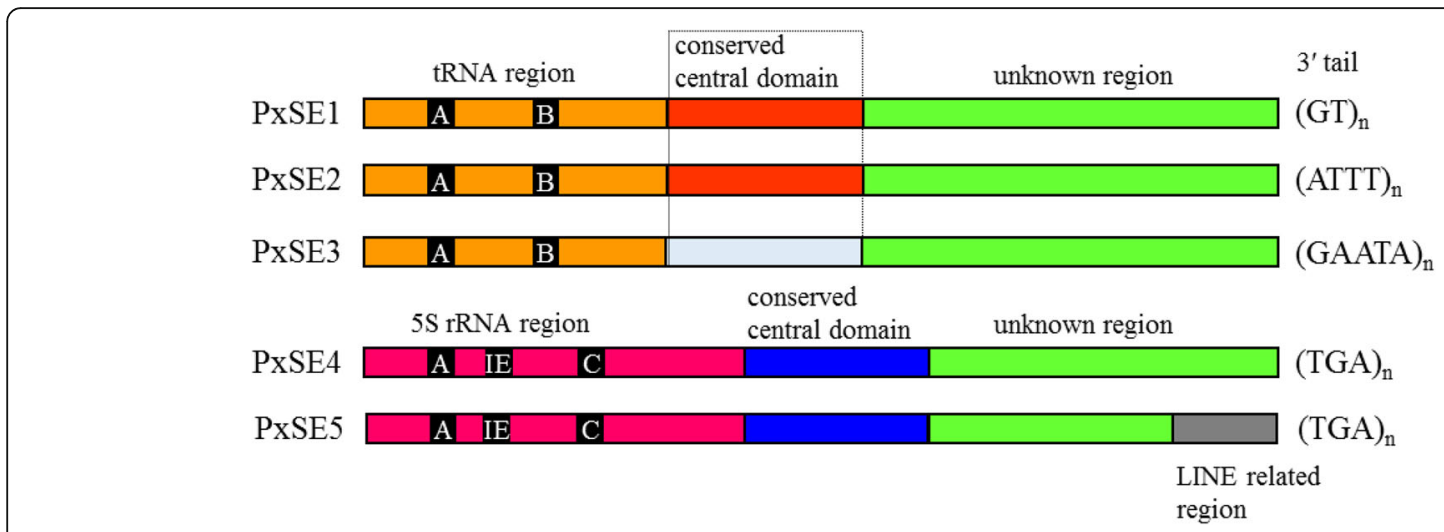

Fig. 1 The schematic representation of structure of PXSE1, PXSE2, PXSE3, PXSE4 and PXSE5 in P. xylostella. The A, B, IE and C in tRNA Arg Or 5S rRNA region represent $\mathrm{A}$ box, $\mathrm{B}$ box, intermediate element and $\mathrm{C}$ box, respectively.The PXSE1, PXSE2 and PXSE3 are tRNA-derived SINES, PXSE4 and PXSE5 are 5 S rRNA-derived SINES

SINE2-1_Pxu from Papilio xuthus [34] (Figs. 1 and 2b). The copy numbers of PXSE2 and PXSE3 were 5056 and 5158 in DBM genome, respectively (Table 1 ). The average divergence of PxSE2 and PxSE3 were 0.071 and 0.089 , respectively (Table 1 ).

\section{Distribution of PXSE1, PXSE2 and PXSE3 in other species}

BLAST searches were performed to detect PXSE1, PXSE2 and PxSE3 sequences in insect species other than $P$. xylostella. In total, homologous sequences of PXSE1, PXSE2 and PXSE3 were identified in five, two and seven Lepidoptera insects, respectively (Accession numbers: MW068230-MW069451, Additional file 2: Figure S2), among which MSSE2 in Manduca sexta showed the highest copy numbers of 16,157, whereas only 533 copies of CSSE1 were detected in the genome of Chilo suppressalis (Table 1). The consensus sequences of these elements vary in size from $252 \mathrm{bp}$ to $333 \mathrm{bp}$ and have different 3 '-tails. Differently, the consensus sequences of EpSE1 did not contain poly(A), poly(T) or simple sequence repeats at 3 '-end. The average divergence varied from 0.035 to 0.13 (Table 1). Although PxSE2- and PxSE3-like elements were not identified in non-insect species, a PXSE1-like element, SINPVSE1, was detected in Spodoptera litura nucleopolyhedrovirus II (EU780426.1: 30485-30735), which was located within ORF27 encoding an unknown protein.

Multiple sequence alignment of the consensus sequences showed that the evolutionary divergence varied from 0.003 to 0.436 . The highest identity (99.7\%) was observed between PMSE1 in Papilio machaon and PzSE1 in Papilio zelicaon, whereas MsSE1 in M. sexta and CfSE1 in Choristoneura fumiferana showed the highest evolutionary divergence (0.436) (Additional file 4: Figure S4).
Two 5S rRNA-derived SINEs, PXSE4 and PXSE5, in $P$. xylostella and related species

Using HaSE3 as a query [33], BLAST searches revealed two 5S rRNA-derived SINEs, PxSE4 and PxSE5, in DBM (Accession numbers: MW068157-MW068229, Figs. 1 and 2c). The boundary of PxSE4 and PxSE5 was further defined by the alignment of single PxSE element and its empty site sequence (Additional file 2: Figure S2 and Figure S3). PXSE4 and PxSE5 are both $389 \mathrm{bp}$ in length and shared high identity of $250 \mathrm{bp}$ sequence at $5^{\prime}$-end but are different at the 3 '-end. The promoter regions of PXSE4 and PXSE5 include the specific A box, IE and C boxes, and shared about 63\% identity with $5 \mathrm{~S}$ rRNA of Bombyx mori, indicating that they are 5S rRNA-derived SINEs (Fig. 2c). The copy numbers and average divergence of PxSE4 and PxSE5 were 4415 and 1952, 0.078 and 0.132 , respectively (Table 1 ).

Interestingly, we found a LINE element PxLINE1.1 (NW_011952036.1: 552486-555,713) with its 43-bp 3'end being $84 \%$ identical to that of PxSE5 (Fig. 2). Thus, this region was designated as 3'-LINE-related region (Fig. 1). The PxLINE1.1 element was $3228 \mathrm{bp}$ long, flanked by 13 bp target site duplications (TSDs), encoded L1_EN (Endonuclease domain of the non-LTR retrotransposon LINE-1) and RT domain, and was terminated by ATGT tetranucleotide repeats in the short 3' untranslated region (3' UTR) (Fig. 3). Additional eight copies were found to be 96.1 to $99.7 \%$ identical to PxLINE1.1 in P. xylostella. Specifically, one copy (AHIO01028576.1:13049_14357) from WGS was inserted as a $1686 \mathrm{bp}$ fragment, which shared $71.8 \%$ identity with mariner-8_BM from B. mori [35] (Table 2 and Additional file 5: Figure S5). Sequences sharing 63 to $82 \%$ identity with the $1580 \mathrm{bp}$ fragments at the 3 '-end of PxLINE1.1 were also found in the other 7 lepidopteran insect genomes (Additional file 6: Figure S6). 
A

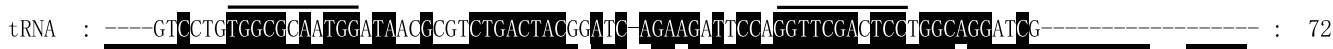
PxSE1 : GACGACCGAATGGCGTAGTGGTTAGTGACCCTGACTACTGAGC-CGATGGTCCCGGGTTCGATTCCCGGCTGGGGCAGATATTTGT---TTAAAC : 91 PxSE2 : GACGACCGAATGGCGTAGTGGTTAGTGGCCCTGACTGCTATGC-CGAAGGTCCCGGGTTCGATTCCCGGCTGGGGCAGATATTTGT---TTAAAG : 91

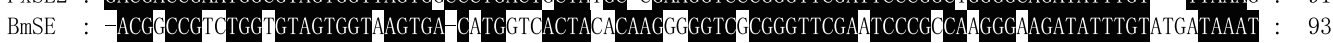

tRNA

PXSE1 : PxSE2

BmSE

tRNA

PxSE1

PxSE2 :

BmSE

B

tRNA

PxSE3

SINE2-1_PXu

ACAGATATTTGTTCTCGGGTCTTGGATGT--GCCCGTAAAATGGCAATAGGCCCGCCCCCTATTACATTGGGACTAACATAACACTCTGGCGAAA : 184 ACAGATATTTGTACTCGGGTCTTGGGTGTTGATATTTATATTTAGTATCTATCTATCTAT----GTATTTGTGTAGATATA----TCAGCTGTCC: 178 ATAAATGTCTTTTCCAGGGTTATGGATG TATATTAAATATATGTATGTGTATAATAAAATCTTACATTTATT----TCCGTTATCT : 176

AGTGGGTGCAGCAATGCACCTCTGCCTACCCCGCAAGGGAGTACATTAGTACAAGGCGTGAG--_--_-_---_GCGTGTGTGTGTGTGT : 263 GACACCCATAACACAGGTTCT----_GCCTAGCTTGGGGTCGGAT--_GGCCGTGTGTGAGATGTCCCCACATATTTATTTATTTATTTATTT : 263 GGTACCTGTAACACAAGTTCTTTACGAACTTATCACGGGACCAGTT---AACGTGGCGTGAT-TGTTAGTAAATATTTATTTATTTATTTATTT : 266

tRNA $\quad$ : PxSE3 : $:$ T-- TTTCTGAATT--TAAGTACAATGTATACCATCGCTCTTACGGTGAAGGAAAACATCGTGAGGAAACCTGCATATCT--_--- : 164 SINE2-1_PXu : TTCGATTTCCGATTTACATATGTAC-ATTTAT-CCGACGTTCTTACGGTGAAGGAAAACATCGTGATGCAACCTGCACATATCTGAGAAG : 171

$\begin{array}{lll}\text { tRNA } & : & \text { PxSE3 }\end{array}$

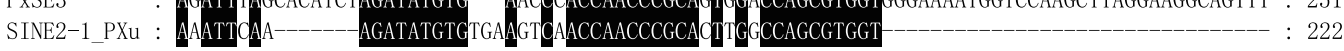

$\begin{array}{llll}\text { tRNA } & : & - \\ \text { PxSE3 } & : & \text { AGACCTTGGGGATATGCACAAAGGTTCCATTCGAGAGAGCCAGGTGCAGGTACTGTTACCCCCACAGAGAATAGAATAGAATAGAATA }: & -\end{array}$

C

5S_rRNA : GCCAACGTCCATACDACGTTGAAAACACCGGTTCTCGT-_CCGATCACGGAAGTCAAGCAACGTCGGGCGTAGTCAGTACTTGGATGGGTGACD : 92 HaSE3 : AGAAGAGCTCTTGGCTAAGTCAAAGCCGCGCGGATCG-ATCGATCA-TAAGGTTAAGCAACGCTTGGCGCGGTCGGTCCGTGGATGGGTGACO : 91 PxSE4 : -GAAGCGTCCGTAGTCGAGC-GGGCCTCAGTGATCGTAACTGATCGCTGAGGTTAAGCAACAACTGACACGGTCAGCCATTGGATGGGTGACO : 91 PxSE5 : -GAAGCGTCCGTAGTCGAGC-GGGCTTCAGTGATCGTAACTGATCACTGAGGTTAAGCAACAACTGACACGGTCAGCCATTGGATGGGTGACO : 91

PxLINE1

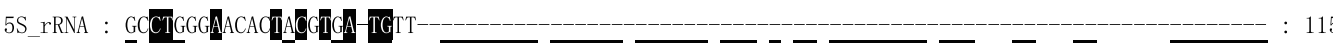
HaSE3 : ATCTT-----GTCATAATGAGTTCTTCCGTGTTTCGGANGGCACGATAAACTGTAGGTCCCGGCTGCCATTTGAACATCTTTGGCAGTCGT : 177 PxSE4 : AATTTCAAGTGGTGCTTTTCTGGACGCTTCCGTGCTTCGGACGGCACGTTAAGCCGTGGGTCCCGGTTGCTGCTTCGGCA--_--GCAGTCGT : 179 PxSE5 : GATTTCAAGTGGTTCTTTTCTGGACGCTTCCGTGCTTCGGACGGCACGTTAAGCCGTGGGTCCCGGTTGCTGCTTCGGCA------GCAGTCGT : 179

PxLTNE1

5S_rRNA :

HaSE3

PxSE4

PxSE5

PxLINE1

TACGGGTAGTCAGAAGCC---AGAAAGTCTGACAACCA TAAGCCTAGTCAGAGGCCTTCGGGCGGCTTGAAAAC TAAGCCTAGTCAGAGGCCTTCGGGCGGCTTGAAAAC

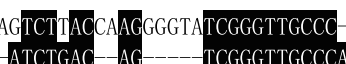
ATCTGAC-

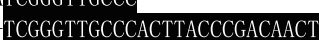

TCTCAGCACAAGC : 264 TCGGGTTGCCCACTTACCCGACAACTTGCATTGTACTCAT : 264

5S_rRNA :

HaSE3

PxSE4

PxSE5

PxLINE1

TTGC---TTGTGTTGGGGTCCACCAACCCGCACTTGGCCAGCGTGGTGGACTAGGCCTAAACCCTTCCTTCATTGGAAGGAGACCCGTGCCCCA CTCTGCCTAC :

5S_rRNA

HaSE3

PxSE4

PxSE5

: 389

PxLINE1 : CCCTCTGGGAG-ACAGGCGTGAGTATATGTATGT : 43

Fig. 2 (See legend on next page.) 
(See figure on previous page.)

Fig. 2 The consensus sequences of PXSE1, PXSE2, PXSE3, PXSE4 and PXSE5. BMSEm, SINE2-1_PXU and HaSE3 sequences were obtained from Repbase database. tRNA and $5 S$ rRNA sequences were downloaded from D. melanogaster tRNA Arg sequence (Accession number: V00243) and B. mori (Accession number: K03316), respectively. a PXSE1 and PXSE2 consensus sequences aligned with tRNA sequence and BmSE. Nucleotides shaded in black are conserved across sequences. The underlined sequences of A Box and B Box are the RNA pol III promoter sequences. $\mathbf{b}$ PXSE3 consensus sequence aligned with tRNA-related region and conserved central domain of SINE2-1_PXU. c PXSE4, PXSE5 consensus sequences aligned with 5S rRNA and 3'-region of PxLINE1.1. PXLINE1.1 is a new LINE transposon in P. xylostella

The PxSE4 and PXSE5 sequences were used as queries to search against the whole genome shotgun (WGS) and expressed sequence tags (EST) database using BLASTN. Three elements, LaSE2, CSSE2 and ObSE2, with high identities to PxSE4 were found in genomes of Lerema accius, C. suppressalis and Operophtera brumata, respectively (Accession numbers: MW068230-MW069451, Additional file 2: Figure S2). In particular, the 115-bp fragment at $5^{\prime}$-end of ObSE2 is different from PxSE4, whereas the central 122-bp fragment shares highly identity with PXSE4 (Additional file 4: Figure S4G and Additional file 7: Figure S7B). The 75-bp fragment at $5^{\prime}$-end of ObSE2 is $54.2 \%$ identical to the 72-bp tRNA of $D$. melanogaster, but different from PxSE1 (Additional file
7: Figure S7A). However, no simple repeat sequences were found at the 3 '-ends of the ObSE2. While we did not find PXSE5-like elements in other insects, the 56-bp fragment at $3{ }^{\prime}$-end of PXSE5 and SfSE1 shared $89.6 \%$ identity (Additional file 4: Figure S4I).

\section{Transpositional burst of SINEs}

Due to the accumulation of random mutations over time, evolutionarily ancient SINE families have a lower sequence identity among copies, whereas SINEs families with recent or ongoing transposition harbor relatively homogeneous copies [12]. To evaluate the periods of transpositional activity and relative age of SINE copies per family of SINEs, we performed a pairwise

Table 1 Novel SINE elements identified in this study

\begin{tabular}{|c|c|c|c|c|c|c|c|}
\hline SINE Family & Species & RNA Origin & Consensus Length & Tail & Copy Number ${ }^{a}$ & Divergence & Resource \\
\hline PXSE1 & Plutella xylostella/Yponomeutoidea & tRNA & 263 & $(\mathrm{GT}) \mathrm{n}$ & $6208(68)$ & 0.035 & WGS \\
\hline MsSE1 & Manduca sexta/Bombycoidea & tRNA & 267 & $(\mathrm{GT}) \mathrm{n}$ & $7513(133)$ & 0.091 & WGS \\
\hline SfSE1 & Spodoptera frugiperda/Noctuoidea & tRNA & 298 & $(\mathrm{ATGT}) \mathrm{n}$ & $11,117(79)$ & 0.130 & WGS \\
\hline SINPVSE1 & Spodoptera litura nucleopolyhedrovirus ॥ & tRNA & 260 & $(T G T T A) n$ & $1(1)$ & ND & $\mathrm{Nr} / \mathrm{nt}$ \\
\hline SlituSE1 & Spodoptera litura/Noctuoidea & tRNA & 259 & (ATGTT)n & $\mathrm{ND}(8)$ & ND & EST \\
\hline SlittSE1 & Spodoptera littoralis/Noctuoidea & tRNA & 270 & $(\mathrm{ATGTT}) \mathrm{n}$ & $\mathrm{ND}(15)$ & ND & EST \\
\hline CfSE1 & Choristoneura fumiferana/Tortricoidea & tRNA & 252 & $(\mathrm{~A} T T) \mathrm{n}$ & $\mathrm{ND}(10)$ & ND & EST \\
\hline PXSE2 & P. xylostella & tRNA & 263 & $(\mathrm{~A} T \mathrm{~T}) \mathrm{n}$ & $5056(33)$ & 0.071 & WGS \\
\hline ObSE1 & Operophtera brumata/Geometroidea & tRNA & 275 & $(\mathrm{TATT}) \mathrm{n}$ & $4521(120)$ & 0.066 & WGS \\
\hline CsSE1 & Chilo suppressalis/Pyraloidea & tRNA & 287 & $(\mathrm{TATT}) \mathrm{n}$ & $533(125)$ & 0.036 & WGS \\
\hline PXSE3 & P. xylostella & tRNA & 339 & $($ GAATA)n & $5158(50)$ & 0.089 & WGS \\
\hline MsSE2 & M. sexta & tRNA & 333 & $(T A T) n$ & $16,157(126)$ & 0.090 & WGS \\
\hline PgSE1 & Papilio glaucus/Papilionoidea & tRNA & 303 & $(T A T) n$ & $1751(66)$ & 0.097 & WGS \\
\hline PmSE1 & Papilio machaon/Papilionoidea & tRNA & 310 & $(G A T) n$ & $5740(138)$ & 0.098 & WGS \\
\hline LaSE1 & Lerema accius/Hesperioidea & tRNA & 319 & $(G A T) n$ & $3832(52)$ & 0.107 & WGS \\
\hline PzSE1 & Papilio zelicaon/Papilionoidea & tRNA & 293 & $(G A T) n$ & $\mathrm{ND}(15)$ & ND & TSA \\
\hline EpSE1 & Erynnis propertius/Hesperioidea & tRNA & 300 & ND & $\mathrm{ND}(8)$ & ND & TSA \\
\hline SeSE1 & Spodoptera exigua/Noctuidae & tRNA & 291 & $(G A T) n$ & $\mathrm{ND}(43)$ & ND & TSA \\
\hline PXSE4 & P. xylostella & $5 S$ rRNA & 389 & $(T G A) n$ & $4415(50)$ & 0.078 & WGS \\
\hline LaSE2 & L. accius & $5 S$ rRNA & 348 & ND & $1214(45)$ & 0.101 & WGS \\
\hline CsSE2 & C. suppressalis & $5 S$ rRNA & 294 & $(T G A) n$ & $532(169)$ & 0.021 & WGS \\
\hline ObSE2 & O. brumata & tRNA & 255 & $(C G A A A) n$ & $863(65)$ & 0.012 & WGS \\
\hline PXSE5 & P. xylostella & $5 S$ rRNA & 389 & $(\mathrm{ATGT}) \mathrm{n}$ & $1952(23)$ & 0.132 & WGS \\
\hline
\end{tabular}

$N D$ not determined

${ }^{a}$ the number in bracket is the number of copies used to reconstruct the consensus sequences 
1 ctctgttgttgtttttggtaacgcAATTGTTTTTCATCTCTCAGTGCTCCGGCCCCTCCCGTGAGTAGCGGTACACCATTCATGGTGCCG

91 TGAAACCGATAACTACACTCCAAGÄGAACAGGGTTCÄATATGGATCAACCCGGTGGTGGCGACGCGGTTCGCAACCCGTCACGCCGTGTC $\begin{array}{llllllllllllllllllllll} & \text { D } & Q & P & G & G & G & D & A & V & R & N & P & S & R & R & V\end{array}$

181 TCTCGGAGGAGCGAAAAGTGGGCTGTGGAGACCCGCATAGCCACGATGAATATTTGTGGGGGTCTGGATGGAAAGATAGATGAAGTATGC $\begin{array}{llllllllllllllllllllllllllllll}S & R & R & S & E & K & W & A & V & E & T & R & I & A & T & M & N & I & C & G & G & L & D & G & K & I & D & E & V & C\end{array}$

271 GAGGTGTTTAACACAAGAGGAATGGATTTGCTATGTGTGAATGAGTCCAAGCGGAAAGGTAGGGGAATTACCACGCACGGAACCTTGACA $\begin{array}{clllllllllllllllllllllllllllll}\mathrm{E} & \mathrm{V} & \mathrm{F} & \mathrm{N} & \mathrm{T} & \mathrm{R} & \mathrm{G} & \mathrm{M} & \mathrm{D} & \mathrm{L} & \mathrm{L} & \mathrm{C} & \mathrm{V} & \mathrm{N} & \mathrm{E} & \mathrm{S} & \mathrm{K} & \mathrm{R} & \mathrm{K} & \mathrm{G} & \mathrm{R} & \mathrm{G} & \mathrm{I} & \mathrm{T} & \mathrm{T} & \mathrm{H} & \mathrm{G} & \mathrm{T} & \mathrm{L} & \mathrm{T}\end{array}$

361 GCCTATTGGTCAGGGGTTCCTGAATCTGAGCATGGCTGTCAAGGGGTTGGGATTGTCCTATCTGAACGCTGGAATAATTGTGTGAAAGAA $\begin{array}{llllllllllllllllllllllllllllll}A & Y & W & S & G & V & P & E & S & E & H & G & C & Q & G & V & G & I & V & L & S & E & R & W & N & N & C & V & K & E\end{array}$

451 TACGAGTGCGTGAGCCCAAGACTTATCTGGATCCGGTTAAAAGTAGGTTTGACGAGATTGTTTGTTCTGGGGGTTTATGCCCCGGACACG

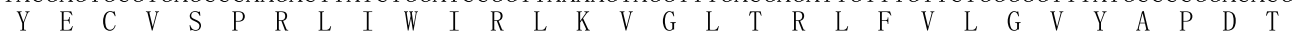

541 TCAAAATCTGCACAGGAGATAGATGAGTTTTGGAAAAGTATGAATGTAGTGTTGGATGAATGCGATGAGAATGAAAGGGTAATTATGCTC $\begin{array}{llllllllllllllllllllllllllllll}S & K & S & A & Q & E & I & D & E & F & W & K & S & M & N & V & V & L & D & E & C & D & E & N & E & R & V & I & M & L\end{array}$

631 GGTGATTTTAATAGTTGGGTAGGTGTGCAGCGTGATGGGTATGAAAGTGTATTGGGTAAGTTTGGTGACGTAAGAGTGAATGAGAATGGC $\begin{array}{llllllllllllllllllllllllllllll}G & D & F & N & S & W & V & G & V & Q & R & D & G & Y & E & S & V & L & G & K & F & G & D & V & R & V & N & E & N & G\end{array}$

721 AGGTATCTCCTGGATGTTTGCTTAGAAAGAAACCTTATAGTTTCTAATTCTATGTTCAGGCACAAGGAGATACATATGTATACATGGCAA

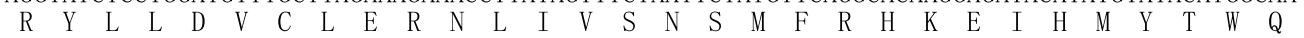

811 CGACAGAATGAAAGGAGCATAATAGACTTTGTAATTGTGGATGAAAGGTTACGCGTAAACGTTGCTGATACTCGAGTCTATCGGGGTGTC $\begin{array}{llllllllllllllllllllllllllllll}R & Q & N & E & R & S & I & I & D & F & V & I & V & D & E & R & L & R & V & N & V & A & D & T & R & V & Y & R & G & V\end{array}$

901 AATGTTGGAACGGACCATTTTCTGGTTGAAAGCCGGATTCGTGGTCTGTTTAATTATTGGCGACACCGACCTCGAGTATCAACAACAAAT $\begin{array}{llllllllllllllllllllllllllllll}\mathrm{N} & \mathrm{V} & G & \mathrm{~T} & \mathrm{D} & \mathrm{H} & \mathrm{F} & \mathrm{L} & \mathrm{V} & \mathrm{E} & \mathrm{S} & \mathrm{R} & \mathrm{I} & \mathrm{R} & \mathrm{G} & \mathrm{L} & \mathrm{F} & \mathrm{N} & \mathrm{Y} & \mathrm{W} & \mathrm{R} & \mathrm{H} & \mathrm{R} & \mathrm{P} & \mathrm{R} & \mathrm{V} & \mathrm{S} & \mathrm{T} & \mathrm{T} & \mathrm{N}\end{array}$

991 TTAGAGCGTATCAAAGTAGAAAAATTGCAAGATGATGTTGTAAGTGAGGAGTATAGAAGAAGTTTGAAAGATAATTTAGAGAGCACTATT

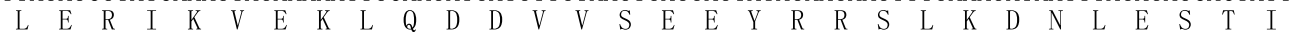

1081 GTCTTGGATGAAAATGATTTAGAAGAAAATTGGAAGCGCTTGAAAGATAGTTTAGTAAATACGGCGGTGAAAGTATGTGGAGTGAATAAA

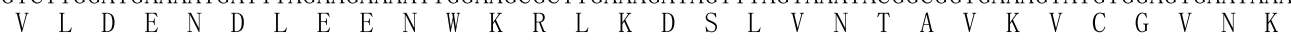

1171 AGAAAGAAGAGTGGTAAGAGGGAGCTTACATGGTGGGATGATGAATGCAAAAAGGTAGTGAATGAAAAGAAAATGGCATGGTTGGATTTT $\begin{array}{llllllllllllllllllllllllllllllllll}R & K & K & S & G & K & R & E & L & T & W & W & D & D & E & C & K & K & V & V & N & E & K & K & M & A & W & L & D & F\end{array}$

1261 TTGTCTAAAAAAGCCAACAACAGAATGCAAGGAAATCAGGGAATGGACGATGAAATGAAAGAGATTCGAGAAAAGTATGTTCTGCTAAAG $\begin{array}{lllllllllllllllllllllllllllllll}\mathrm{L} & \mathrm{S} & \mathrm{K} & \mathrm{K} & \mathrm{A} & \mathrm{N} & \mathrm{N} & \mathrm{R} & \mathrm{M} & \mathrm{Q} & \mathrm{G} & \mathrm{N} & \mathrm{Q} & \mathrm{G} & \mathrm{M} & \mathrm{D} & \mathrm{D} & \mathrm{E} & \mathrm{M} & \mathrm{K} & \mathrm{E} & \mathrm{I} & \mathrm{R} & \mathrm{E} & \mathrm{K} & \mathrm{Y} & \mathrm{V} & \mathrm{L} & \mathrm{L} & \mathrm{K}\end{array}$

1351 AAAAAAGTAAAAGAAGTGATTGAAAGAAAGAAGAAGGCGTTAAAAGATGAATATGACAGGAAATTCTCTGACAACTTTCGAGCGAACATT $\begin{array}{llllllllllllllllllllllllllllllll}K & K & V & K & E & V & I & E & R & K & K & K & A & L & K & D & E & Y & D & R & K & F & S & D & N & F & R & A & N & I \\ \end{array}$

1441 AAATTGTTCTGGAAGTTGGTAAGAAAGGCTCGAGGGAAGTCAGAGAATACAAATCTGGATGTGATAAGGGATGAAAATGGAGATGTTTTG

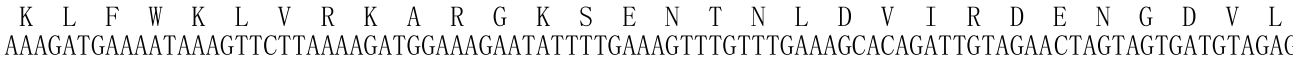
$\begin{array}{lllllllllllllllllllllllllllllll}K & D & E & N & K & V & L & K & R & W & K & E & Y & F & E & S & L & F & E & S & T & D & C & R & T & S & S & D & V & E\end{array}$

1621 GTAGAAAATGATAGAATAGTAGATGAGGAAAACAAGATAAGTATGAAAGAAATTATGGAAGCATTAAAAAGAATGAAAGTTGGTAAATCG \begin{tabular}{llllllllllllllllllllllllllllll}
$V$ & $E$ & $N$ & $D$ & $R$ & $I$ & $V$ & $D$ & $E$ & $E$ & $N$ & $K$ & $I$ & $S$ & $M$ & $K$ & $E$ & $I$ & $M$ & $E$ & $A$ & $L$ & $K$ & $R$ & $M$ & $K$ & $V$ & $G$ & $K$ & $S$ \\
\hline
\end{tabular}

1711 GCCGGGTATGATAGGGTATCTTTAGAGATGCTAAGAGCGGGAGGTGGAGTGGCTGCAAGTGAGCTTTACCAACTCTTCAATAAGTGCTGG

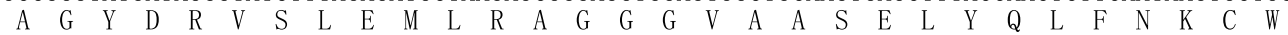

1801 CGTTGCGGTACTGTGCCACGCGACTGGTGCAGAGCGGTCATAGTTCCTTTGTATAAAGGAAAAGGCTCGCTCCAGACCTGCAACAGTTAC $\begin{array}{lllllllllllllllllllllllllllllll}R & C & G & T & V & P & R & D & W & C & R & A & V & I & V & P & L & Y & K & G & K & G & S & L & Q & T & C & N & S & Y\end{array}$

1891 CGCGGCATCAGCCTCTTGAGTATAGTTGGTAAATTGTATGCAAAAATATTGATTGAAAGGGTAGTGAAAGAGACGGAAGAAAAGATCTGG $\begin{array}{llllllllllllllllllllllllllllll}R & G & I & S & L & L & S & I & V & G & K & L & Y & A & K & I & L & I & E & R & V & V & K & E & T & E & E & K & I & W \\ \end{array}$

1981 GATGTGCAAGGTGGTTTTCGAAAGGGGATGGGATGTACGGATCAAGTCTTTTCTCTACGAAGCGTCACAGAAAAGGTCCTTGCAAAGCAG $\begin{array}{llllllllllllllllllllllllllllll}\mathrm{D} & \mathrm{V} & \mathrm{Q} & \mathrm{G} & \mathrm{G} & \mathrm{F} & \mathrm{R} & \mathrm{K} & \mathrm{G} & \mathrm{M} & \mathrm{G} & \mathrm{C} & \mathrm{T} & \mathrm{D} & \mathrm{Q} & \mathrm{V} & \mathrm{F} & \mathrm{S} & \mathrm{L} & \mathrm{R} & \mathrm{S} & \mathrm{V} & \mathrm{T} & \mathrm{E} & \mathrm{K} & \mathrm{V} & \mathrm{L} & \mathrm{A} & \mathrm{K} & \mathrm{Q}\end{array}$

2071 CAAAAGGTTTTTTGTGCCTTCGTAGATTTGGAAAAGGCTTATGATAGAGTGAGGAGGAATGAATTATGGGAGACACTGTCCGTTTATGGA $\begin{array}{llllllllllllllllllllllllllllll}Q & K & V & F & C & A & F & V & D & L & E & K & A & Y & D & R & V & R & R & N & E & L & W & E & T & L & S & V & Y & G\end{array}$ 2161 GTGGACAGTCACCTGACGCGGGCACTGGGGTCCCTTTATAGGGAGTCTAGCGCTTGTGTCAGGATAAACGGAGCCTACACGGACTGGTTT $\begin{array}{llllllllllllllllllllllllllllll}V & D & S & H & L & T & R & A & L & G & S & L & Y & R & E & S & S & A & C & V & R & I & N & G & A & Y & T & D & W & F\end{array}$ 2251 GATATCCACAGGGGTGTTAGACAGGGATGTGTGGCTTCGCCTTGGCTGTTTAATCTGTTTATGGATAGTTGTCTCAAGGATATGAAAGAT $\begin{array}{llllllllllllllllllllllllllllll}D & I & H & R & G & V & R & Q & G & C & V & A & S & P & W & L & F & N & L & F & M & D & S & C & L & K & D & M & K & D\end{array}$ 2341 GATGAAAGAGGTTTACGAATAGGAGAGTTACTTCTTAAGTGCTTGCTGTATGCTGACGATCAAGTCATACTTGCATCGTCGGTAGAACAG $\begin{array}{llllllllllllllllllllllllllllll}\mathrm{D} & \mathrm{E} & \mathrm{R} & \mathrm{G} & \mathrm{L} & \mathrm{R} & \mathrm{I} & \mathrm{G} & \mathrm{E} & \mathrm{L} & \mathrm{L} & \mathrm{L} & \mathrm{K} & \mathrm{C} & \mathrm{L} & \mathrm{L} & \mathrm{Y} & \mathrm{A} & \mathrm{D} & \mathrm{D} & \mathrm{Q} & \mathrm{V} & \mathrm{I} & \mathrm{L} & \mathrm{A} & \mathrm{S} & \mathrm{S} & \mathrm{V} & \mathrm{E} & \mathrm{Q}\end{array}$ 2431 CTGCAACAACAAGTAACTCTCATGCATGAAAGTTTTAAAAGGAAAGGAATGAAAGTGAATGTTAGTAAGACGAAAGTGATGGTGTTTGAA $\begin{array}{llllllllllllllllllllllllllllll}L & Q & Q & Q & V & T & L & M & H & E & S & F & K & R & K & G & M & K & V & N & V & S & K & T & K & V & M & V & F & E \\ \end{array}$ 2521 AGAGATGAAGAGGTAACTGAATGTGAGATCACGATCGAGAACGAAAGAGTGGAGCAAGTGAATGAGTTTGTATATTTGGGTAGTCTGTTT $\begin{array}{llllllllllllllllllllllllllllllll}R & D & E & E & V & T & E & C & E & I & T & I & E & N & E & R & V & E & Q & V & N & E & F & V & Y & L & G & S & L & F\end{array}$ 2611 ACGAGGGATGGGAAATGTGAGGGGGATATTGAAAGAAGAGTGAAAGCGGGAAATAAAGTGAATGGGGCCTTGCACTCTTTCATGGGGAGT $\begin{array}{llllllllllllllllllllllllllllllll}T & R & D & G & K & C & E & G & D & I & E & R & R & V & K & A & G & N & K & V & N & G & A & L & H & S & F & M & G & S\end{array}$ 2701 CAAAGCGTGTCTCAAAAGGCTCGTTTGGCTGTTCATGGGGGAGTGTTGGTCCCTACGCTAATGTATGGAAGTGAAAGCTGGGTCTGGCAG $\begin{array}{lllllllllllllllllllllllllllllll}Q & S & V & S & Q & K & A & R & L & A & V & H & G & G & V & L & V & P & T & L & M & Y & G & S & E & S & W & V & W & Q\end{array}$

2791 AAGAAGAATGAAAGTAGGGTAAATGCTGTTGAGATGCGCTCTCTAAGAAGTATGTGTGGACTGAAACTGAATGATAAGATAAGGAATAGT

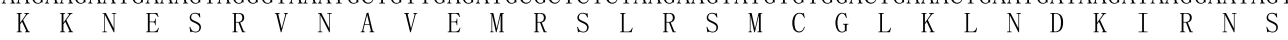
2881 ATTATAAGAGAGCGAGTTGGAGTAAAAGAAGACGTAGTGACCAAAATTGAAAAAGGAATGTTGAGATGGTTTGGTCACATTGAAAGGATG $\begin{array}{llllllllllllllllllllllllllllllll}I & I & R & E & R & V & G & V & K & E & D & V & V & T & K & I & E & K & G & M & L & R & W & F & G & H & I & E & R & M\end{array}$ 2971 GATGAAAGAAGACTGACGAAAGAAATTTACTGTGCAGAGATGAATGGTTGTGTCGGTAGAGGACGTCCTAGGCGAAAGTATGTCGACCAG

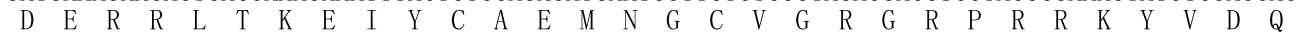
3061 ATAGGCGACATACTCAAGAAGAGCCAAATTAGGAGTTTCCGAAACCGACGTGCGTGTATGAAGAGACTAATGAATGTTGAGGAAGCGAAA $\begin{array}{cccccccccccccccccccccccccccccc}\text { I } & G & D & I & L & K & K & S & Q & I & R & S & F & R & N & R & R & A & C & M & K & R & L & M & N & V & E & E & A & K\end{array}$ 3151 GAAGTATGTCAGGATCGTGGCACGTGGAGATCCATAGTCTCTGCCTACCCCTCTGGGAGACAGGCGTGAGTATATGTATGTATGTATGTA 3241 ATTGTTTTTCATacaaaaattgtctgtccctcggag

Fig. 3 The nucleotide sequence and conceptual translation of the partner LINE element, PXLINE1.1, for PXSE5. Flanking direct repeats are indicated in lowercase. The nucleotides of TSD are indicated with the wavy line. The nucleotides of 3' tail sequence are indicated with the straight line 
Table 2 Copies with high identity to PxLINE1.1 in P. xylostella

\begin{tabular}{|c|c|c|c|c|c|c|}
\hline Subject No. & Identity \% & Length & Start & End & Evalue & Bit score \\
\hline $\mathrm{AHIO01036046.1}$ & 99.6 & 3203 & 31,532 & 34,758 & 0 & 5838 \\
\hline AHIO01028688.1 & 99.5 & 3203 & 1034 & 4236 & 0 & 5819 \\
\hline AHIO01028673.1 & 99.4 & 3203 & 10,866 & 7665 & 0 & 5810 \\
\hline AHIO01016682.1 & 99.3 & 3203 & 4885 & 8087 & 0 & 5797 \\
\hline AHIO01031207.1 & 99.2 & 3207 & 51 & 3252 & 0 & 5784 \\
\hline AHIO01033561.1 & 99 & 3209 & 7965 & 4750 & 0 & 5749 \\
\hline AHIO01003557.1 & 96.1 & 3214 & 43,696 & 40,488 & 0 & 5243 \\
\hline AHIO01028576.1 & 99.7 & 4408 & 11,375 & 15,783 & 0 & 5067 \\
\hline
\end{tabular}

comparison of SINE copies with the consensus sequences of respective family and grouped them into intervals from 80 to $100 \%$ identity. As shown in Fig. 4, 4796 of 6208 copies of PxSE1 show more than 95\% identity to the consensus sequence, of which 223 copies are $100 \%$ identical to PXSE1 consensus sequence (Additional file 8: Table S1), indicating a recent transpositional burst. A strong transposition peak with high identity values is also observed in PxSE2, PxSE3, and PXSE4. However, PXSE5 shows high numbers of diverged copies, and only 49 copies (2.5\%) of PxSE5 have more than $95 \%$ identity with its consensus sequence (Fig. 4).

The activity profiles deduced from similarity intervals of SINEs in other lepidopteran species revealed a recent transpositional burst of CSSE1 and CSSE2 in C. suppressalis and ObSE2 in O. brumata, whereas LaSE1 and SFSE1 harbour diverged copies and only few young ones (Fig. 4 and Additional file 9: Figure S8). High number of copies with a wide range of identity values were observed in MsSE1, MsSE2, PgSE1, PmSE1 and LaSE2 (Fig. 4 and Additional file 9: Figure S8). Due to few copies in related EST and transcriptome shotgun assembly (TSA) databases, the distribution profiles of copy identity in SlituSE1, SlittSE1, CfSE1, PzSE1, EpSE1 and SeSE1 were not subject to analysis.

\section{Contribution of SINEs to gene and genome evolution in P. xylostella}

The integration pattern relative to the annotated genes in the genome of P. xylostella was analyzed. A total of

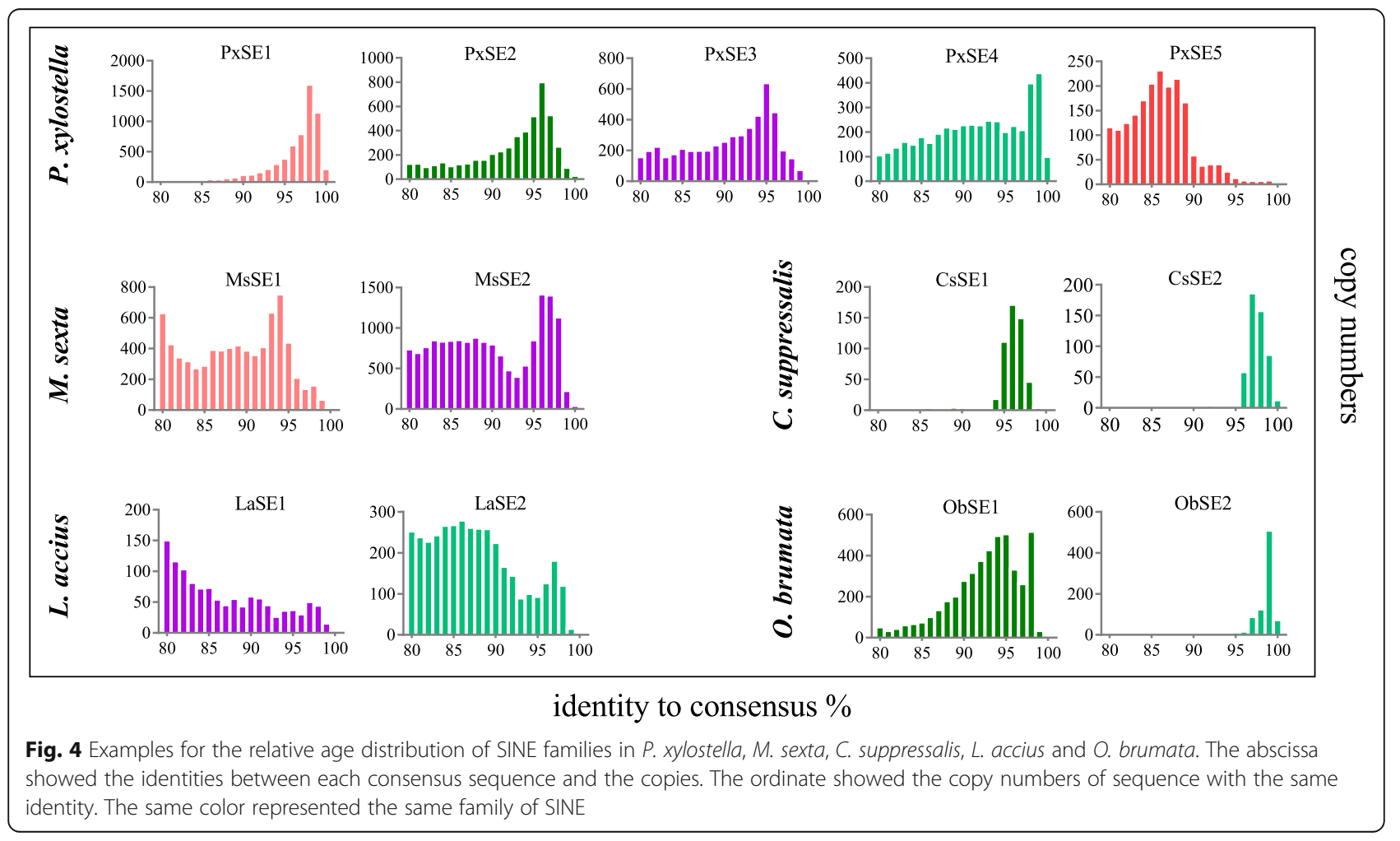


2750 out of 6208 copies (44\%) of PXSE1, 2478 out of 5056 copies (49\%) of PxSE2, 2470 out of 5158 copies (48\%) of PxSE3, 2265 out of 4415 copies (51\%) of PXSE4 and 902 out of 1952 copies (46\%) of PXSE5 were found in introns (Fig. 5a). Similar proportions of the copies are distributed in regions $5 \mathrm{kbp}$ downstream of genes. Only two, five, five, eight and five copies of PxSE1, PxSE2, PXSE3, PxSE4 and PXSE5 were found to insert into exonic regions, respectively (Fig. 5a). Among them, 11 copies are inserted into the coding regions (CDS), a copy is inserted into the $5^{\prime}$ UTR, and 13 copies are inserted into the 3' UTR (Table 3). Most of these genes were annotated as enzymes or enzyme-associated proteins, and were related to signal transduction, splicing, metabolism. For example, a 261 bp copy PXSE2.2 of PXSE2 family from DBM genome (NW_011952011.1: 22733562273095) inserted into CDS of a gene encoding nitrogen permease regulator 3 -like protein. The 21-bp fragment at 5 '-end of PXSE2.2 contributed 7 amino acids to the $\mathrm{N}$-terminus of the protein (Fig. $5 \mathrm{~b}$ ).

Further analysis revealed the insertion of multiple copies of SINE families into introns of the same gene. As many as 60 elements inserted into introns of LOC105382892 gene, including 18, 14, 10, 11 and 7 copies of PXSE1, PXSE2, PXSE3, PXSE4 and PXSE5, respectively (Additional file 10: Figure S9). A total of 95 genes were found to be inserted with at least ten copies of
SINE elements (Additional file 10: Figure S9D). Thus, the P. xylostella SINE families contribute to structural variation in introns, which might influence the regulation of gene expression.

\section{Evolution and horizontal transposon transfer (HTT) of SINES}

The phylogenetic tree of the 23 SINE consensus sequences showed that the SINEs with the same internal Pol III promoter were clustered together, except ObSE2 SINE (Fig. 6a). Due to the high identity of PXSE1 and $P x S E 2$ at 5 '-ends, the clustering of related SINEs in different family, such as PxSE1, PxSE2, CfSE1, ObSE1 and CSSE1, is not surprising. The comparison of phylogenetic tree of PXSE3 family and the taxonomy tree of related host species [36, 37] (Fig. 6) suggests some degree of vertical transmission of PXSE3 family in lepidopteran insects. Interestingly, SINPVSE1 and SfSE1 in Spodoptera frugiperda, SlittSE1 in Spodoptera littoralis and SlituSE1 in S. litura, were clustered together (Table 1 and Fig. 6a). The orthologous outer flanking sequence of SINPVSE1 were identified in Spodoptera eridania nucleopolyhedrovirus isolate 251 and Spodoptera cosmioides nucleopolyhedrovirus isolate VPN72, suggesting that SINPVSE1 inserted into the genome of nucleopolyhedrovirus by HTT. In addition, the inter 5 '-flanking sequence (about $800 \mathrm{bp}$ ) was found to share $95 \%$ identity

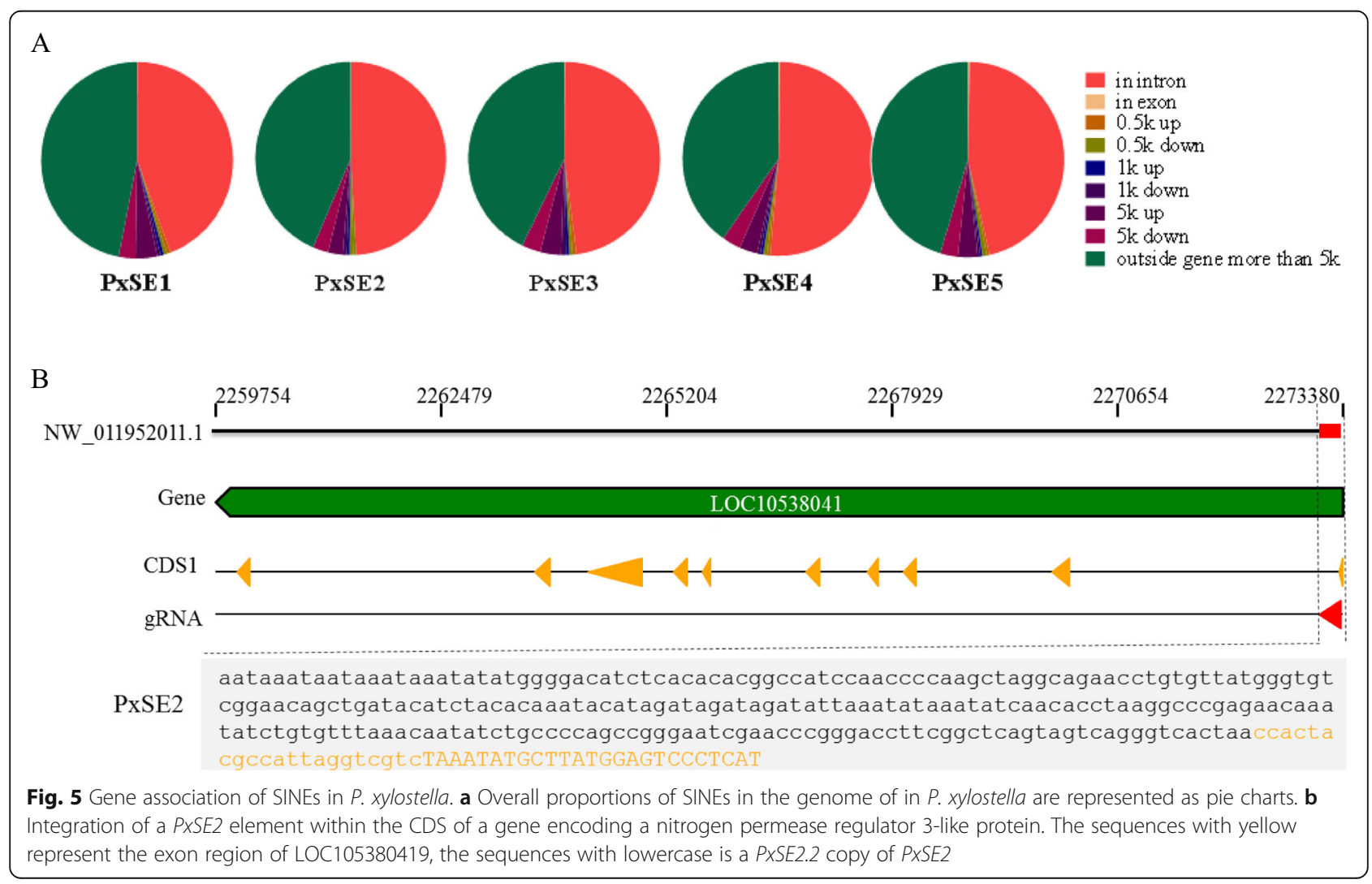


Table 3 The annotation of SINEs copies integrated into CDS and untranslated regions (UTR) in P. xylostella

\begin{tabular}{|c|c|c|c|c|c|c|}
\hline Copies & Location & GenelD & $\begin{array}{l}\text { Gene } \\
\text { mapping }\end{array}$ & $\begin{array}{l}\text { COG class } \\
\text { annotation }\end{array}$ & Swissprot annotation & $\mathrm{Nr}$ annotation \\
\hline PXSE1.1 & $\begin{array}{l}436,420- \\
436,680\end{array}$ & LOC105383591 & CDS & $\begin{array}{l}\text { Signal transduction } \\
\text { mechanisms }\end{array}$ & $\begin{array}{l}\text { Cyclic nucleotide-gated cation } \\
\text { channel subunit A }\end{array}$ & uncharacterized protein \\
\hline PXSE1.2 & $\begin{array}{l}46,181-46 \\
441\end{array}$ & LOC105394666 & CDS & $\begin{array}{l}\text { Signal transduction } \\
\text { mechanisms }\end{array}$ & $\begin{array}{l}\text { Cyclic nucleotide-gated cation } \\
\text { channel subunit A }\end{array}$ & uncharacterized protein \\
\hline PXSE2.1 & $\begin{array}{l}800,671- \\
800,788\end{array}$ & LOC105384210 & 3' UTR & ND & Transcription factor 25 homolog & transcription factor 25 \\
\hline PXSE2.2 & $\begin{array}{l}2,273,356-2 \\
273,095\end{array}$ & LOC105380419 & CDS & ND & $\begin{array}{l}\text { Nitrogen permease regulator 3- } \\
\text { like protein }\end{array}$ & $\begin{array}{l}\text { nitrogen permease regulator 3-like } \\
\text { protein }\end{array}$ \\
\hline PXSE2.3 & $\begin{array}{l}35,405-35 \\
544\end{array}$ & LOC105390425 & 3' UTR & ND & Serine/threonine-protein kinase & $\begin{array}{l}\text { serine/threonine-protein kinase grp- } \\
\text { like }\end{array}$ \\
\hline PXSE2.4 & $\begin{array}{l}250,511- \\
250,756\end{array}$ & LOC105381765 & CDS & $\begin{array}{l}\text { Replication, } \\
\text { recombination and } \\
\text { repair }\end{array}$ & DNA topoisomerase 3-alpha & uncharacterized protein \\
\hline PXSE2.5 & $\begin{array}{l}266,277- \\
266,424\end{array}$ & LOC105391817 & 3' UTR & ND & $\begin{array}{l}\text { Leucine-rich repeat serine/ } \\
\text { threonine-protein kinase } 1\end{array}$ & uncharacterized protein \\
\hline PXSE3.1 & $\begin{array}{l}274,387- \\
274,287\end{array}$ & LOC105388973 & 3' UTR & ND & $\begin{array}{l}\text { Gamete and mating-type specific } \\
\text { protein A }\end{array}$ & uncharacterized protein \\
\hline PXSE3.2 & $\begin{array}{l}699,462- \\
699,145\end{array}$ & LOC105386775 & CDS & ND & Uncharacterized protein & $\begin{array}{l}\text { serine/arginine repetitive matrix } \\
\text { protein } 1 \text {-like }\end{array}$ \\
\hline PXSE3.3 & $\begin{array}{l}490,234- \\
490,329\end{array}$ & LOC105381296 & 3' UTR & ND & $\begin{array}{l}\text { Venom acid phosphatase Acph-1 } \\
\text { (Precursor) }\end{array}$ & prostatic acid phosphatase \\
\hline PXSE3.4 & $\begin{array}{l}149,472- \\
149,747\end{array}$ & LOC105389005 & 3' UTR & $\begin{array}{l}\text { General function } \\
\text { prediction only }\end{array}$ & Protein suppressor of hairy wing & zinc finger protein 26-like \\
\hline PXSE3.5 & $\begin{array}{l}165,558- \\
165,422\end{array}$ & LOC105383366 & CDS & ND & $\begin{array}{l}\text { Mediator of RNA polymerase II } \\
\text { transcription subunit } 12\end{array}$ & uncharacterized protein \\
\hline PXSE4.1 & $\begin{array}{l}1,260,998-1 \\
260,894\end{array}$ & LOC105380733 & 3' UTR & $\begin{array}{l}\text { General function } \\
\text { prediction only }\end{array}$ & Ras-related protein Rab-24 & ras-related protein Rab-24-like \\
\hline PXSE4.2 & $\begin{array}{l}63,533-63 \\
636\end{array}$ & LOC105391976 & 3' UTR & $\begin{array}{l}\text { General function } \\
\text { prediction only }\end{array}$ & Protein fem-1 homolog B & protein fem-1 homolog B \\
\hline PXSE4.3 & $\begin{array}{l}31,764-31 \\
620\end{array}$ & LOC105393953 & 3' UTR & $\begin{array}{l}\text { General function } \\
\text { prediction only }\end{array}$ & Ras-related protein Rab-24 & ras-related protein Rab-24-like \\
\hline PXSE4.4 & $\begin{array}{l}787,511- \\
787,311\end{array}$ & LOC105382052 & 3' UTR & $\begin{array}{l}\text { RNA processing and } \\
\text { modification }\end{array}$ & $\begin{array}{l}\text { WW domain-containing protein } \\
\text { ZK1098.1 }\end{array}$ & $\begin{array}{l}\text { transcription elongation regulator 1- } \\
\text { like isoform } X 1\end{array}$ \\
\hline PXSE4.5 & $\begin{array}{l}160,917- \\
160,809\end{array}$ & LOC105388357 & CDS & ND & Serine/threonine kinase SAD-1 & $\begin{array}{l}\text { PAS domain-containing serine/threo- } \\
\text { nine-protein kinase }\end{array}$ \\
\hline PXSE4.6 & $\begin{array}{l}1,160,410-1 \\
160,304\end{array}$ & LOC105398290 & 3' UTR & $\begin{array}{l}\text { General function } \\
\text { prediction only }\end{array}$ & Ras-related protein Rab-24 & ras-related protein Rab-24-like \\
\hline PXSE4.7 & $\begin{array}{l}819,669- \\
819,771\end{array}$ & LOC105385258 & CDS & ND & $\begin{array}{l}\text { Probable 4-coumarate-CoA ligase } \\
2\end{array}$ & probable 4-coumarate-CoA ligase 3 \\
\hline PXSE4.8 & $\begin{array}{l}217,956- \\
218,125\end{array}$ & LOC105383715 & CDS & ND & $\begin{array}{l}\text { Integrin alpha-PS3 light chain } \\
\text { (Precursor) }\end{array}$ & integrin alpha-PS5-like \\
\hline PXSE5.1 & $\begin{array}{l}306,942- \\
307,066\end{array}$ & LOC105398401 & 5' UTR & ND & ND & $\begin{array}{l}\text { CLK4-associating serine/arginine rich } \\
\text { protein-like }\end{array}$ \\
\hline PXSE5.2 & $\begin{array}{l}101,368- \\
101,480\end{array}$ & LOC105393324 & CDS & $\begin{array}{l}\text { Coenzyme transport } \\
\text { and metabolism }\end{array}$ & $\begin{array}{l}\text { Molybdopterin synthase catalytic } \\
\text { subunit }\end{array}$ & $\begin{array}{l}\text { molybdopterin synthase catalytic } \\
\text { subunit-like }\end{array}$ \\
\hline PXSE5.3 & $\begin{array}{l}993,048- \\
992,940\end{array}$ & LOC105383883 & CDS & ND & $\begin{array}{l}\text { Salivary glue protein Sgs-3 } \\
\text { (Precursor) }\end{array}$ & uncharacterized protein \\
\hline PXSE5.4 & $\begin{array}{l}338,901- \\
338,769\end{array}$ & LOC105387600 & 3' UTR & ND & Alpha-(1,3)-fucosyltransferase C & alpha-(1,3)-fucosyltransferase C-like \\
\hline PXSE5.5 & $\begin{array}{l}499,158- \\
499,032\end{array}$ & LOC105388075 & 3' UTR & ND & Cuticle collagen 1 (Precursor) & $\begin{array}{l}\text { breast cancer metastasis-suppressor } \\
1 \text {-like protein isoform } X 1\end{array}$ \\
\hline
\end{tabular}




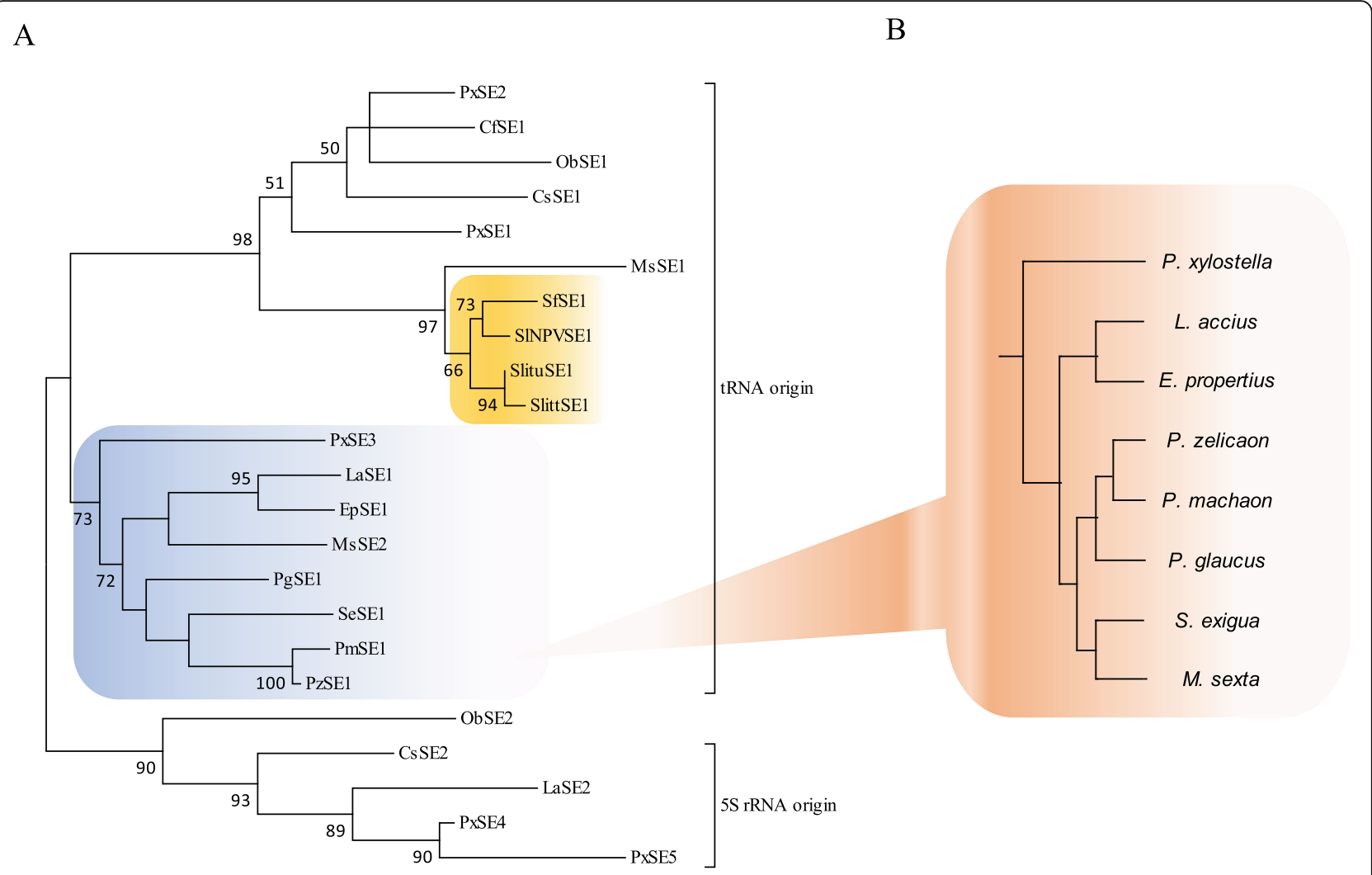

Fig. 6 The evolutionary tree of 23 novel SINEs in this study (a) and the taxonomy tree of lepidopteran insects harboring PXSE3-like SINES (b)

to the sequence (WNNL01000005.1: 248783-248238) of Spodoptera exigua genome (Additional file 11: Figure S10 and Fig. 7), putatively resulted from unknown horizontal gene transfer.

\section{Discussion}

The structure of three tRNA-derived and two 5S rRNAderived SINE families

Up to now, more than 234 SINEs have been isolated from the genomes of human, mammals, reptiles, fishes, mollusks, fungus, green plants, and insects [2]. Based on current data, the tRNA-derived SINEs $(\sim 84 \%)$ were found widely in eukaryotic genomes [2]. Apart from the $5^{\prime}$ terminal head, SINEs also consist of typical body and variable repeated tail. In this study, we have identified three tRNA-derived SINE families, PxSE1, PxSE2 and PXSE3. The $45 \mathrm{bp}$ region in body region of PXSE1 and PXSE2 also showed high identity (93.3\%) except the highly identical heads. Similarly, two $5 \mathrm{~S}$ rRNA-derived SINEs, PxSE4 and PxSE5, also shared $98.7 \%$ identity in $159 \mathrm{bp}$ region of their bodies. Previous studies have found that the conserved bodies of SINE mainly include the V-domain, CORE-domain, Deu-domain, Nindomain, Ceph-domain, Inv-domain, Pln-domain, Snaildomain, and Meta-domain [38-43]. However, the body regions identified in PXSEs are different from these known domains. A hypothesis has proposed that nonautonomous LINEs that have only $5^{\prime}$ and $3^{\prime}$ regions of original LINEs can be a source of enigmatic middle body of SINEs [1]. Hence, highly identical conserved central domains among different SINEs in the same species suggests that the conserved central domain may originated from the same LINE family and has been under strong selective constraint, which is important for reverse transcription. In addition, despite the high identity between ObSE2 and 5S rRNA-derived PxSE4, ObSE2 is a tRNAderived SINE.

\section{Partner LINE}

SINEs can be composed of $5^{\prime}$ and $3^{\prime}$ regions of nonautonomous LINEs, and their $3^{\prime}$ tails will also exchange with other LINEs under the pressure of natural selection to facilitate rapid amplification [1]. The tail homologous to LINE is important for SINE, which allows the integration of new copies of SINE into the new genomic locations using the LINE RT [44]. LINE RT can specifically recognize the 3' homologous SINE tails, indicated that SINE can be mobilized by the retrotransposition machinery of a partner LINE [45]. Here, nine novel LINE copies in P. xylostella, and seven LINEs in each of the lepidopteran insects were identified with 3 '-end similar to that of PXSE5 and SfSE1, suggesting that the LINE 


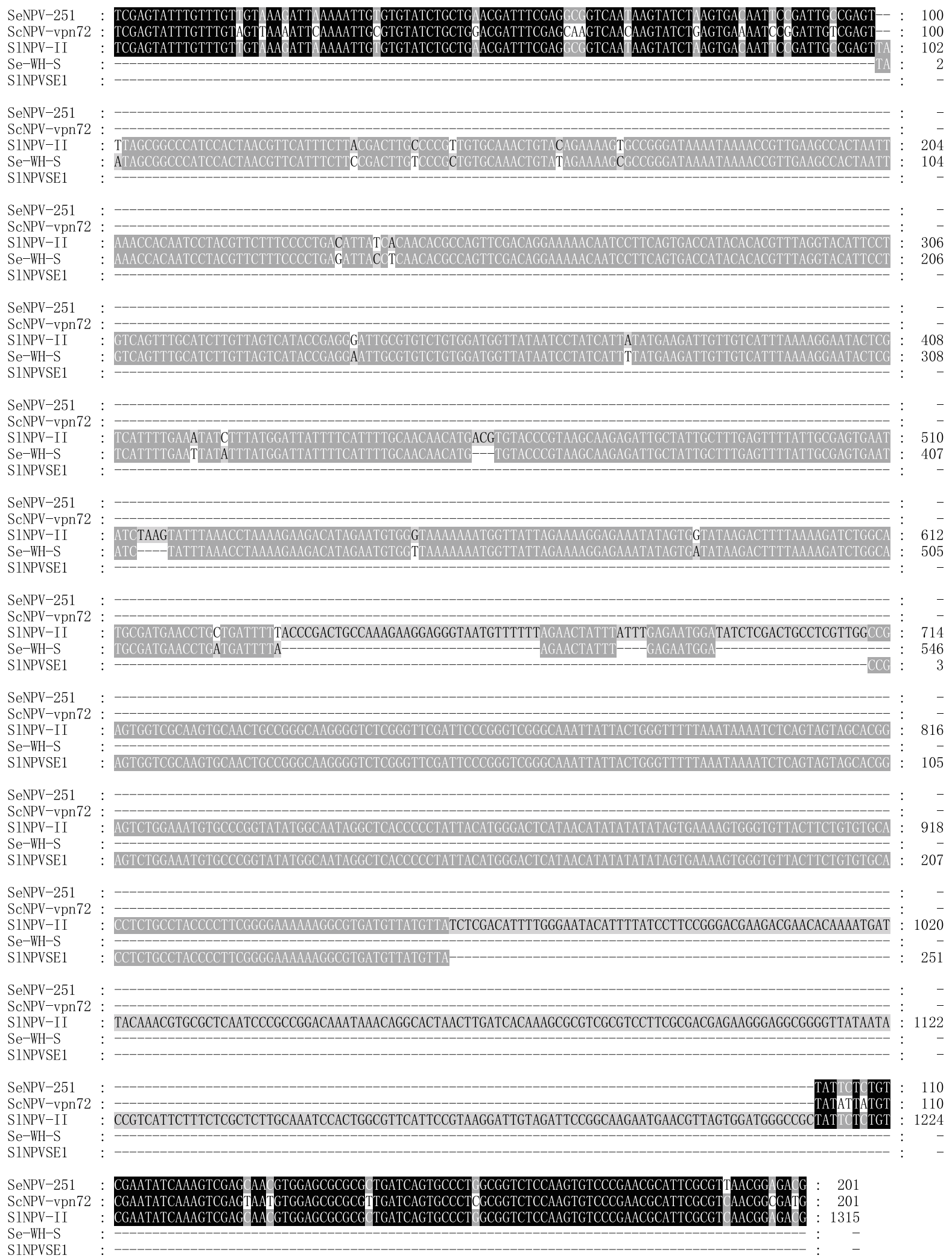

Fig. 7 (See legend on next page.) 
(See figure on previous page.)

Fig. 7 The evidence of HTT from Lepidoptera to baculovirus. Multiple sequence alignment of SINPVSE1 and its flanking sequences and the orthologous sequences. Se-WH-S is a host sequence from S. exigua genome (WNNL01000005.1:248783-248238), SINPV-II is baculovirus sequence from S. litura nucleopolyhedrovirus II (Accession number: EU780426.1:29774-31088) containing SINE copy, SeNPV-251 and ScNPV-vpn72 are orthologous sequecnes of SINPV-II from S. eridania nucleopolyhedrovirus isolate 251 (Accession number: MH320559.1:31479-31679) and S. cosmioides nucleopolyhedrovirus isolate VPN72 (Accession number: MK419955.1:32601-32796), respectively

identified in this study is an ancient retrotransposon and might widely exist in Lepidoptera insects. However, the $5^{\prime}$ regions among SfSE1, PXSE5 and PXLINE1 shared a large divergence, indicating these SINEs exploded after the exchange of 3 '-tails. Moreover, the distinct 3 ' -end in other PXSES suggested that these SINEs might be mobilized by other LINEs that were not identified yet.

\section{Relative age and distribution of SINEs in Lepidoptera insect}

The copy numbers of SINEs varies among different families and species. In P. xylostella, the copy numbers of SINEs of tRNA origin is relatively higher than that of $5 \mathrm{~S}$ rRNA origin. In particular, the copy numbers of PxSE5 is only 1952. Previously, it was speculated that the type 1 promoter in $5 \mathrm{~S}$ rRNAs is more dependent on upstream signals than the type 2 promoter in tRNAs, resulting in the Pol III promoter in a retroposed 5S rRNA copy presumably remains silent or is expressed at a low level [5]. In different species, the copy numbers of the same origin SINE is different. The copy numbers of MSSE1 and MsSE2 in M. sexta and SfSE1 in S. frugiperda were 7513, 16,157 and 11,117 , respectively, whereas only 4521 copies of ObSE1 and 863 copies of ObSE2 were found in $O$. brumata. The genome sizes of $M$. sexta and S. frugiperda are around $400 \mathrm{Mb}$, while O. brumata has larger genome size of $618 \mathrm{Mb}$. Hence, SINE copy number may not correlate with genome size. Some factors of 3 '-tail, such as poly(A) tail or short direct repeats length, sequence conservation and distance to the transcriptional terminator, may affect the retroposition efficiency of the SINE families [46, 47]. In this study, the varied 3'-tail of these SINEs in different species may have affected their distribution in the genome. However, their relationship with the number of copies cannot be determined at this time.

Based on the divergence of the copies from the consensus sequence, the relative age distribution of identified SINEs was analyzed. Scattered age profiles were found in most SINEs among all species or within the genus, suggesting that the activity and accumulation of these SINEs are dynamic processes that can vary considerably between host lineages and SINE lineages. Especially, the highly identity and concentrated PxSE1 showed that it most likely is a relatively young retransposon in the genome of P. xylostella and was generated by recent explosive amplification. The scattered distribution of PXSE5 copies also suggests that it is older than other SINEs.

\section{SINEs contribute to DBM genome evolution}

The ability of TEs to replicate and move in the genome affects the genomic structure, gene expression, and the divergence and evolution of host species [48-51]. The genome size of DBM is $343.575 \mathrm{Mb}$, of which the intronic region occupies of $35.23 \%(121.039 \mathrm{Mb})$ [52]. The integration pattern analysis revealed that the numbers of PxSINEs inserted into introns accounted for 44-51\%, only 2-8 copies were inserted into exons, indicating that PxSINEs prefer to insert or accumulate in introns of genic regions. However, the proportions of different SINEs located within introns of Solanaceae range from 15 to 54\% [53] and 96\% of SINEs inside genes were located inside introns in Zoysia japonica and maize [54], suggesting that the distribution characteristics of SINEs varied in different species. Introns have long been an exemplar of regulated splicing, which affects and enhances almost every step of mRNA metabolism by the act of their removal [55]. In mice, a recent insertion of MT-C retrotransposon into DICER intron truncated its first 6 exons, providing an alternative promoter and a novel first exon. This change resulted in acquizition of oocytespecific expression and is essential for fertility [56]. We speculate that the insertions of PxSEs into introns may provide signals for alternative splicing and polyadenylation, which may be a reflection of the host response to an ever-changing environment.

Importantly, we also noticed that only 25 copies of SINEs inserted into the genic exonsof DBM, of which 13 copies were found in 3' UTR. In eukaryotic cells, some proteins (such as PUF protein) can bind to regulatory elements in the 3' UTR of mRNAs and control mRNA stability, translation and localization [57]. The genes with the insertion of SINEs into exons are mainly annotated in terms of metabolism, cell division, signal transduction and transportation, and it remains to be elucidated whether some of the SINE insertions have an influence on gene expression.

\section{HTT of SINEs}

Increasing evidence showed that HTT is a common phenomenon. So far, no less than 5689 HTT events have been recorded [58]. However, only a few HTT events of 
SINE have been detected, including the SmaI-cor SINE between coregonid and common ancestor of salmonid (Hamada et al. 1997), Sauria SINE between reptiles and mammals [59], HaSE2 SINE between Aphis gossypii and Lepidoptera insects [33]. The long-term vertical inheritance property inherent in SINE and its dependence on active partner LINEs to move in new hosts may be the reason why HTT events rarely occur [47, 60], as was confirmed by the partial congruence between the phylogenetic trees of PxSE3 families and host species in this study. Interestingly, SINPVSE1, a SINE copy inserted into the baculovirus, shared more than $90 \%$ identity to the consensus sequence of SFSE1, SlittSE1 and SlituSE1 (Additional file 4: Figure S4B). In addition, the absence of target site duplication as well as upstream host sequence in SINPV-II, suggested that non-homologous end-joining of double-strand breaks might be the mechanism of HTT. SINPV can successfully infect $S$. litura and S. exigua [61]. S. exigua multicapsid nucleopolyhedrovirus (SeMNPV) DNA can also replicate in five nonpermissive cell lines including SF21AEII, CLS-79, SpLi221, hi-5 and $\mathrm{BmN} 4$ [62], indicating a wider host range of NPV. Thus, our finding suggests the occurrence of HTT of PXSE1 between baculovirus and Lepidoptera insects. This is not surprising, because population genomics supported baculoviruses as vectors of horizontal transfer of insect transposons [63]. Similarly, the HTT of Helitron transposon $\mathrm{Hel}-2$ and Tc1-like transposon $T C p 3.2$ between insects and associated baculoviruses has been detected $[64,65]$. Recent studies have revealed that the occurrence of HTT generally exhibits species ecological relationships, such as host-parasite $[66,67]$ and predator-prey $[68,69]$. Additionaly, proviruses have been reported as vectors for HTT of Sauria SINE from reptiles to mammals [59]. Hence, it is necessary to further explore the HTT events of PxSE1-like elements mediated by baculoviruses.

\section{Conclusions}

In this study, we identified three tRNA-derived SINEs and two 5S RNA-derived SINEs in the genome of $P$. xylostella, among which PXSE1 is a relatively young retrotransposon and was generated by recent explosive amplification. Homology searches revealed scattered distribution of these elements in other Lepidopteran insects with variable copy numbers. The preference of PxSINEs to insert or accumulate in introns of genic regions indicated that P. xylostella SINE families contribute to structural variation in introns. The identification of PxSE1-like elements in the baculovirus and related lepidopteran host insects provides evidence of horizontal transfer facilitated by host-parasite interactions. These data may have implications for understanding the evolution and HT mechanisms of SINEs.

\section{Methods}

\section{Data resources}

The 235 publicly available insect databases of WGS assemblies including 33 Lepidoptera insects, EST, nucleotide $(\mathrm{Nr} / \mathrm{Nt})$, and TSA from National Center for Biotechnology Information (NCBI) (last accessed November 30, 2018) were used in this study (Additional file 12: Table S2). P. xylostella WGS was downloaded from NCBI [52]. As corresponding gene annotation file, the GFF files GCF_000330985.1 were used.

\section{Database search strategy}

To identify SINE candidates, database searches were performed and composed of four steps. Firstly, the known SINE sequences, including tRNA-derived HaSE1 from Helicoverpa armigera [33] and BmSE from B mori [28], $5 S$ rRNA-derived HaSE3 from $H$. armigera [33], were used as queries for local blastn in the DBM genome. The sequences of high homology (at least $70 \%$ identity over at least $50 \mathrm{bp}$ length to query) as well as $500 \mathrm{bp}$ upstream and downstream flanking regions were extracted using TBtools [70] and analyzed for conserved structural motifs of SINEs such as internal RNA Pol III promoter and TSDs. The consensus sequences of PXSE1 and PXSE4 were determined by multiple sequence alignments. Secondly, the consensus sequences of PxSE1 and PXSE4 were searched against DBM genome by local BLASTN to identify other potential homologous sequences, and two other tRNA-derived PxSE2 and PXSE3 and a 5S rRNA-related PXSE5 were identified. Thirdly, the 50-bp fragment at 3 '-end of SINE families was used as query to search potential partner LINEs, and the LINE, PXLINE1, related to PXSE5 was identified. Finally, insect genome databases as well as EST, $\mathrm{Nr} / \mathrm{Nt}$ and TSA databases from NCBI were searched using consensus sequences of these five SINE families as queries to detect SINEs in species other than DBM.

\section{Copy number estimation}

To estimate copy number and average divergence of SINEs, respective consensus sequences were used to search against related databases (Additional file 12: Table S2). All contiguous sequences with at least $80 \%$ identity at the nucleotide level to the consensus over $100 \mathrm{bp}$ were used to estimate copy number in all species [71, 72]. Given the high sequence identity of $5^{\prime}$-ends in several copies of different SINE families in DBM, all those undistinguishable copies were ruled out. For example, PXSE1 and PXSE2 shared high identity of $120 \mathrm{bp}$ sequence at 5 '-ends, thus all copies aligned only with part or all of this $120 \mathrm{bp}$ region in the consensus sequence were excluded for copy number analysis. Further, all fragments sharing at least $80 \%$ identity over at least $80 \%$ of the length of the consensus sequence were 
aligned and used for calculation of average divergence to consensus sequence with Kimura-2 parameter model [73]. The identity value of single copy to consensus sequence was rounded to an integer for the relative age distribution analysis [53].

\section{Gene association and genomic show cases}

The association of DBM SINEs with annotated genes were investigated using custom Perl script from MapGene2Chrom (http://mg2c.iask.in/mg2c_v1.0) [74]. The integration of SINEs into genic regions including introns, coding and untranslated regions as well as the distances of intergenic copies to the closest neighboring gene were determined as described previously [53]. The number of SINEs within each region was counted and the results were graphically represented using MapGene2Chrom.

\section{Sequence analysis and phylogeny}

SINE's tRNA-like structure was checked with tRNAscan-SE [75], using mixed model and cove score cut off value $=0.01$ as default. Multiple SINE copies were aligned by MUSCLE [76], and the alignments were visualized with GENEDOC (www.psc.edu/biomed/genedoc). The phylogeny of full consensus sequences of SINE families was built by MEGA 7.0 using Maximum Likehood with $\mathrm{K} 2+\mathrm{G}$ model [77]. The reliability of the trees was tested using 1000 bootstrap replications [71].

\section{Abbreviations}

SINEs: Short interspersed nuclear elements; LINEs: Long interspersed nuclear elements; TEs: Transposable elements; RT: Reverse transcriptase;

DBM: Diamondback moth; TSDs: Target site duplications; UTR: Untranslated regions; WGS: Whole genome shotgun; EST: Expressed sequence tags; CDS: Coding regions; HTT: Horizontal transposon transfer; TSA: Transcriptome shotgun assembly; Nr/Nt: Nucleotide; NCBI: National Center for Biotechnology Information

\section{Supplementary Information}

The online version contains supplementary material available at https://doi. org/10.1186/s12864-021-07543-z.

Additional file 1: Figure S1. Characteristic of PXSE1 in P. xylostella. (A) the sequence of PXSE1. The pink nucleotides are TSD sequence, gray background present A box and B box structure, green background is $3^{\prime}$ tail sequence. (B) the homology search of PXSE1 in Repbase database.

Additional file 2: Figure S2. The consensus sequence of tRNA and 5S rRNA related SINE transposons in insect genomes. Nucleotides in red font are $3^{\prime}$ tail sequences.

Additional file 3: Figure S3. Multiple sequence alignment the consensus sequence of PXSE1 (A), PXSE2 (B), PXSE3 (C), PXSE4 (D), PXSE5 (E) and their empty site sequences. The nucleotides of TSD are indicated with the red words. The nucleotides of $3^{\prime}$ tail sequence are indicated with the gray background.

Additional file 4: Figure S4. Multiple sequence alignment and evolutionary divergence estimation between the consensus sequences of SINES. The number of base differences per site from between sequences are shown. All ambiguous positions were removed for each sequence pair. Evolutionary analyses were conducted in MEGA7.0.
Additional file 5: Figure S5. The 8 copies with high identity to PXLINE1.1 in P. xylostella (A) and the multiple sequence alignment of one copy (AHIO01028576.1: 13049_14357) and Mariner-8_BM (B).

Additional file 6: Figure S6. Alignment of potential LINE transposons in 8 lepidopteran insects genome. PXLINE1 in P. xylostella (NW01 1952036.1: 552486-555,713), TaLINE1 in Tuta absoluta (SNMR 01038797.1: 8533-11852), McLINE1 in Melitaea cinxia (APLT01012314.1: 14517-16103), CCLINE1 in Conopomorpha cramerella (SJJU01072145.1: 61771-65266), GmLINE1 in Galleria mellonella (NHTH01000021.1: 42306714228043), ArLINE1 in Adela reaumurella (WYDE01048472.1: 2507-535), AhLINE1 in Adoxophyes honmai (BHDV01006067.1: 48096-49728), DpLINE1 in Dendrolimus punctatus (JAABVI010000027.1: 8196917-8193378).

Additional file 7: Figure S7. The origin analysis of ObSE2. (A) the alignment of 75-bp fragment at 5'-end of ObSE2 and 72-bp tRNA-related region of $D$. melanogaster. (B) the schematic representation of structure of ObSE2.

Additional file 8: Table S1. The copies of PXSE1 in the genome of $P$. xylostella.

Additional file 9: Figure S8. Examples for the relative age distribution of SINE families in other species based on the identity to the speciesspecific consensus. The abscissa showed that the identity between each consensus sequence and the copies. The ordinate showed that the copy numbers of sequence with the same identity.

Additional file 10: Figure S9. The typical integration pattern of SINES within genome of $P$. xylostella. (A) (B) and (C) are schematic diagrams of several copies inserted into the introns of LOC105382892, LOC105381513 and LOC105383359, respectively. (D) Statistics number of different SINE families inserted into the same gene.

Additional file 11: Figure S10. Paralogous empty sites of PXSE1 (A) in P. xylostella and SFSE7 (B) in S. frugiperda. The nucleotides of TSD are indicated with the red background. The nucleotides of $3^{\prime}$ tail sequence are indicated with the gray background.

Additional file 12: Table S2. The databases of NCBI used for Blast searches, including 8 WGS databases, 3 EST databases and 2 TSA databases as well as the $\mathrm{Nr} / \mathrm{nt}$ database.

\section{Acknowledgements}

Not Applicable.

\section{Authors' contributions}

$J W$ and JX conceived and designed the research. GH, NZ, HJ, XM, KQ and YZ analyzed the data. GH and JW wrote the manuscript. All authors read and approved the final manuscript.

\section{Funding}

This work was supported by the National Natural Science Foundation of China (Grant No. 31701792), Jiangsu Agricultural Science and Technology Innovation Fund (Grant No. ZX (17)2002).

\section{Availability of data and materials}

The original sequences used to construct the consensus sequences of all SINEs are uploaded to the NCBI database (http://www.ncbi.nlm.nih.gov/ bioproject/665855).

The complete genome of Plutella xylostella is available at the NCBI RefSeq assembly database under the accession number GCF_000330985.1 and the other lepidopteran genome databases including the accesion numbers are available in Additional file 12: Table S2.

\section{Declarations}

Ethics approval and consent to participate Not applicable.

\section{Consent for publication}

Not applicable.

\section{Competing interests}

The authors declare that they have no competing interests. 


\section{Author details}

${ }^{1}$ College of Horticulture and Plant Protection, Yangzhou University, Yangzhou 225009, China. ${ }^{2}$ Jiangsu Lixiahe District Institute of Agricultural Sciences, Yangzhou 225008, China. ${ }^{3}$ Joint International Research Laboratory of Agriculture andAgri-Product Safety of the Ministry of Education, Yangzhou University, Yangzhou 225009, China.

\section{Received: 3 August 2020 Accepted: 22 March 2021}

\section{Published online: 31 March 2021}

\section{References}

1. Kojima KK. LINEs contribute to the origins of middle bodies of SINEs besides 3' tails. Genome Biol Evol. 2018;10(1):370-9. https://doi.org/10.1093/gbe/evy008.

2. Vassetzky NS, Kramerov DA. SINEBase: a database and tool for SINE analysis. Nucleic Acids Res. 2013;41(Database issue):D83-9. https://doi.org/10.1093/na r/gks1263.

3. Kramerov DA, Vassetzky NS. Short retroposons in eukaryotic genomes. Int Rev Cytol. 2005;247:165-221. https://doi.org/10.1016/S0074-7696(05)47004-7.

4. Ohshima K, Okada N. SINEs and LINEs: symbionts of eukaryotic genomes with a common tail. Cytogenet Genome Res. 2005;110(1-4):475-90. https:// doi.org/10.1159/000084981.

5. Kapitonov W, Jurka J. A novel class of SINE elements derived from 5 S rRNA. Mol Biol Evol. 2003;20(5):694-702. https://doi.org/10.1093/molbev/msg075.

6. Gogolevsky KP, Vassetzky NS, Kramerov DA. 5S rRNA-derived and tRNAderived SINEs in fruit bats. Genomics. 2009;93(5):494-500. https://doi.org/1 0.1016/j.ygeno.2009.02.001

7. Kojima KK. A new class of SINEs with snRNA gene-derived heads. Genome Biol Evol. 2015;7(6):1702-12. https://doi.org/10.1093/gbe/evv100.

8. Longo MS, Brown JD, Zhang C, O'Neill MJ, O'Neill RJ. Identification of a recently active mammalian SINE derived from ribosomal RNA. Genome Biol Evol. 2015;7(3):775-88. https://doi.org/10.1093/gbe/evv015.

9. Suh A, Witt CC, Menger J, Sadanandan KR, Podsiadlowski L, Gerth M, et al. Ancient horizontal transfers of retrotransposons between birds and ancestors of human pathogenic nematodes. Nat Commun. 2016;7(1):1-9.

10. Schramm L, Hernandez N. Recruitment of RNA polymerase III to its target promoters. Genes Dev. 2002;16(20):2593-620. https://doi.org/10.1101/gad.101 8902.

11. Consortium IHGS. Initial sequencing and analysis of the human genome. Nature. 2001;409:860-921.

12. Kogler A, Schmidt T, Wenke T. Evolutionary modes of emergence of short interspersed nuclear element (SINE) families in grasses. Plant J. 2017;92(4): 676-95. https://doi.org/10.1111/tpj.13676.

13. Schwichtenberg K, Wenke T, Zakrzewski F, Seibt KM, Minoche AE, Dohm JC, et al. Diversification, evolution and methylation of short interspersed nuclear element families in sugar beet and related Amaranthaceae species. Plant J. 2016;85(2):229-44. https://doi.org/10.1111/tpj.13103.

14. Nishihara H, Okada N. Retroposons: genetic footprints on the evolutionary paths of life. In: Murphy WJ editor. Methods in molecular biology: phylogenomics. Totowa: Humana Press Inc; 2008. pp. 201-25.

15. Lisch D. How important are transposons for plant evolution? Nat Rev Genet. 2013;14(1):49-61. https://doi.org/10.1038/nrg3374.

16. Trizzino M, Park Y, Holsbach-Beltrame M, Aracena K, Mika K, Caliskan M, et al. Transposable elements are the primary source of novelty in primate gene regulation. Genome Res. 2017;27(10):1623-33. https://doi.org/10.1101/gr.21814 9.116

17. Lev-Maor G, Ram O, Kim E, Sela N, Goren A, Levanon EY, et al. Intronic Alus influence alternative splicing. PLoS Genet. 2008;4(9):e1000204. https://doi. org/10.1371/journal.pgen.1000204.

18. Lee JY, Ji Z, Tian B. Phylogenetic analysis of mRNA polyadenylation sites reveals a role of transposable elements in evolution of the $3^{\prime}$-end of genes. Nucleic Acids Res. 2008;36(17):5581-90. https://doi.org/10.1093/nar/gkn540.

19. Loke JC, Stahlberg EA, Strenski DG, Haas BJ, Wood PC, Li QQ. Compilation of mRNA polyadenylation signals in Arabidopsis revealed a new signal element and potential secondary structures. Plant Physiol. 2005;138(3):1457-68. https://doi.org/10.1104/pp.105.060541.

20. Sorek R, Ast G, Graur D. Alu-containing exons are alternatively spliced. Genome Res. 2002;12(7):1060-7. https://doi.org/10.1101/gr.229302.

21. Volff JN. Turning junk into gold: domestication of transposable elements and the creation of new genes in eukaryotes. Bioessays. 2006;28(9):913-22 https://doi.org/10.1002/bies.20452.
22. Durruthy-Durruthy J, Sebastiano V, Wossidlo M, Cepeda D, Cui J, Grow EJ, et al. The primate-specific noncoding RNA HPAT5 regulates pluripotency during human preimplantation development and nuclear reprogramming. Nat Genet. 2016;48(1):44-52. https://doi.org/10.1038/ng.3449.

23. Deininger P. Alu elements: know the SINEs. Genome Biol. 2011;12(12):236. https://doi.org/10.1186/gb-2011-12-12-236.

24. Luchetti A, Lomiento M, Mantovani B. Riding the Wave. The SINE-specific V highly-conserved domain spread into mammalian genomes exploiting the replication burst of the MER6 DNA transposon. Int J Mol Sci. 2019;20(22):5607.

25. Ben-David S, Yaakov B, Kashkush K. Genome-wide analysis of short interspersed nuclear elements SINES revealed high sequence conservation, gene association and retrotranspositional activity in wheat. Plant J. 2013; 76(2):201-10. https://doi.org/10.1111/tpj.12285.

26. Bao W, Kojima KK, Kohany O. Repbase update, a database of repetitive elements in eukaryotic genomes. Mob DNA. 2015;6(1):11. https://doi.org/1 0.1186/s13100-015-0041-9.

27. Adams DS, Eickbush TH, Herrera RJ, Lizardi PM. A highly reiterated family of transcribed oligo (a)-terminated, interspersed DNA elements in the genome of Bombyx mori. J Mol Biol. 1986;187(4):465-78. https://doi.org/10.1016/ 0022-2836(86)90327-X

28. Xu J, Liu T, Li D, Zhang Z, Xia Q, Zhou Z. BmSE, a SINE family with 3' ends of (ATTT) repeats in domesticated silkworm (Bombyx mori). J Genet Genomics. 2010;37(2):125-35. https:/doi.org/10.1016/S1673-8527(09)60031-X

29. Tu Z. Genomic and evolutionary analysis of Feilai, a diverse family of highly reiterated SINEs in the yellow fever mosquito, Aedes aegypti. Mol Biol Evol. 1999;6(6):760-72.

30. Feschotte C, Fourrier N, Desmons I, Mouches C. Birth of a retroposon: the twin SINE family from the vector mosquito Culex pipiens may have originated from a dimeric tRNA precursor. Mol Biol Evol. 2001;18(1):74-84. https://doi.org/10.1093/oxfordjournals.molbev.a003721.

31. Kapitonov $V$, Jurka J. SINE3-1_TC, a family of SINE3 retrotransposons from the red flour beetle genome. Repbase Rep. 2007;7:1180.

32. Santolamazza F, Mancini E, Simard F, Qi Y, Tu Z, della Torre A. Insertion polymorphisms of SINE200 retrotransposons within speciation islands of Anopheles gambiae molecular forms. Malaria J. 2008;7(1):163.

33. Wang J, Wang A, Han Z, Zhang Z, Li F, Li X. Characterization of three novel SINE families with unusual features in Helicoverpa armigera. PLoS One. 2012; 7(2):e31355. https://doi.org/10.1371/journal.pone.0031355.

34. Kojima KK, Jurka J. SINEs from the Asian swallowtail genome. Repbase Rep. 2015:15(9):3203.

35. Jurka J. DNA transposons from Bombyx mori. Repbase Rep. 2010;10(7):943.

36. Kawahara AY, Plotkin D, Espeland M, Meusemann K, Toussaint EFA, Donath A, et al. Phylogenomics reveals the evolutionary timing and pattern of butterflies and moths. Proc Natl Acad Sci U S A. 2019;116(45):22657-63. https://doi.org/10.1073/pnas.1907847116.

37. Zakharov EV, Caterino MS, Sperling FAH. Molecular phylogeny, historical biogeography, and divergence time estimates for swallowtail butterflies of the genus Papilio (Lepidoptera: Papilionidae). Syst Biol. 2004;53(2):278-98.

38. Nishihara H, Plazzi F, Passamonti M, Okada N. MetaSINEs: broad distribution of a novel SINE superfamily in animals. Genome Biol Evol. 2016;8(3):528-39. https://doi.org/10.1093/gbe/evw029.

39. Akasaki T, Nikaido M, Nishihara H, Tsuchiya K, Segawa S, Okada N. Characterization of a novel SINE superfamily from invertebrates: "CephSINEs" from the genomes of squids and cuttlefish. Gene. 2010;454(1-2):819. https://doi.org/10.1016/j.gene.2009.11.005.

40. Gilbert N, Labuda D. CORE-SINEs: eukaryotic short interspersed retroposing elements with common sequence motifs. Proc Natl Acad Sci U S A. 1999; 96(6):2869-74. https://doi.org/10.1073/pnas.96.6.2869.

41. Ogiwara I, Miya M, Ohshima K, Okada N. V-SINEs: a new superfamily of vertebrate SINEs that are widespread in vertebrate genomes and retain a strongly conserved segment within each repetitive unit. Genome Res. 2002; 12(2):316-24. https://doi.org/10.1101/gr.212302.

42. Nishihara H, Smit AF, Okada N. Functional noncoding sequences derived from SINEs in the mammalian genome. Genome Res. 2006;16(7):864-74. https://doi.org/10.1101/gr.5255506.

43. Matetovici I, Sajgo S, lanc B, Ochis C, Bulzu P, Popescu O, et al. Mobile element evolution playing jigsaw-SINEs in gastropod and bivalve mollusks. Genome Biol Evol. 2016;8(1):253-70. https://doi.org/10.1093/gbe/evv257.

44. Kramerov D, Vassetzky N. Origin and evolution of SINEs in eukaryotic genomes. Heredity. 2011;107(6):487-95. https://doi.org/10.1038/hdy.2011.43. 
45. Kajikawa M, Okada N. LINEs mobilize SINEs in the eel through a shared 3' sequence. Cell. 2002;111(3):433-44. https://doi.org/10.1016/S0092-8674(02)01 041-3.

46. Roy-Engel AM, Salem A-H, Oyeniran OO, Deininger L, Hedges DJ, Kilroy GE, et al. Active Alu element "A-tails": size does matter. Genome Res. 2002;12(9): 1333-44. https://doi.org/10.1101/gr.384802.

47. Comeaux MS, Roy-Engel AM, Hedges DJ, Deininger PL. Diverse cis factors controlling Alu retrotransposition: what causes Alu elements to die? Genome Res. 2009;19(4):545-55. https://doi.org/10.1101/gr.089789.108.

48. Liu D, Yang J, Tang W, Zhang X, Royster CM, Zhang M. SINE retrotransposon variation drives ecotypic disparity in natural populations of Coilia nasus. Mob DNA. 2020;11(1):4. https://doi.org/10.1186/s13100-019-0198-8.

49. Ray DA, Grimshaw JR, Halsey MK, Korstian JM, Osmanski AB, Sullivan KAM, et al. Simultaneous TE analysis of 19 heliconiine butterflies yields novel insights into rapid te-based genome diversification and multiple SINE births and deaths. Genome Biol Evol. 2019;11(8):2162-77. https://doi.org/10.1093/gbe/evz125.

50. Trizzino M, Kapusta A, Brown CD. Transposable elements generate regulatory novelty in a tissue-specific fashion. BMC Genomics. 2018;19(1): 468. https://doi.org/10.1186/s12864-018-4850-3.

51. Huang K, Li CF, Wu J, Wei JH, Zou Y, Han MJ, et al. Enhancer activity of Helitron in sericin-1 gene promoter from Bombyx mori. Insect Sci. 2016;23(3): 396-405. https://doi.org/10.1111/1744-7917.12347.

52. You M, Yue Z, He W, Yang X, Yang G, Xie M, et al. A heterozygous moth genome provides insights into herbivory and detoxification. Nat Genet. 2013;45(2):220-5. https://doi.org/10.1038/ng.2524.

53. Seibt KM, Wenke T, Muders K, Truberg B, Schmidt T. Short interspersed nuclear elements (SINEs) are abundant in Solanaceae and have a familyspecific impact on gene structure and genome organization. Plant J. 2016; 86(3):268-85. https://doi.org/10.1111/tpj.13170.

54. Mao $\mathrm{H}$, Wang $\mathrm{H}$. Distribution, diversity, and long-term retention of grass short interspersed nuclear elements (SINEs). Genome Biol Evol. 2017;9(8): 2048-56. https://doi.org/10.1093/gbe/evx145.

55. Le Hir H, Nott A, Moore MJ. How introns influence and enhance eukaryotic gene expression. Trends Biochem Sci. 2003;28(4):215-20. https://doi.org/10.1 016/S0968-0004(03)00052-5.

56. Flemr M, Malik R, Franke V, Nejepinska J, Sedlacek R, Vlahovicek K, et al. A retrotransposon-driven dicer isoform directs endogenous small interfering RNA production in mouse oocytes. Cell. 2013;155(4):807-16. https://doi. org/10.1016/j.cell.2013.10.001.

57. Wickens M, Bernstein DS, Kimble J, Parker R. A PUF family portrait: 3' UTR regulation as a way of life. Trends Genet. 2002;18(3):150-7. https://doi.org/1 0.1016/S0168-9525(01)02616-6.

58. Dotto BR, Carvalho EL, da Silva AF, Dezordi FZ, Pinto PM. Campos TdL, Rezende AM, Wallau GdL. HTT-DB: new features and updates. Database. 2018;2018. https://doi.org/10.1093/database/bax102.

59. Piskurek O, Okada N. Poxviruses as possible vectors for horizontal transfer of retroposons from reptiles to mammals. Proc Natl Acad Sci U S A. 2007; 104(29):12046-51. https://doi.org/10.1073/pnas.0700531104.

60. Luchetti A, Mantovani B. Rare horizontal transmission does not hide longterm inheritance of SINE highly conserved domains in the metazoan evolution. Curr Zool. 2016;62(6):667-74. https://doi.org/10.1093/cz/zow095.

61. Takatsuka J, Okuno S, Ishii T, Nakai M, Kunimi Y. Host range of two multiple nucleopolyhedroviruses isolated from Spodoptera litura. Biol Control. 2007; 41(2):264-71. https://doi.org/10.1016/j.biocontrol.2007.01.014.

62. Yanase T, Yasunaga C, Kawarabata T. Replication of Spodoptera exigua nucleopolyhedrovirus in permissive and non-permissive lepidopteran cell lines. Acta Virol. 1998;42(5):293-8.

63. Gilbert C, Chateigner A, Ernenwein L, Barbe V, Bézier A, Herniou EA, et al. Population genomics supports baculoviruses as vectors of horizontal transfer of insect transposons. Nat Commun. 2014;5(1):1-9.

64. Jehle JA, Nickel A, Vlak JM, Backhaus H. Horizontal escape of the novel Tc1like lepidopteran transposon TCp3.2 into Cydia pomonella granulovirus. J Mol Evol. 1998;46(2):215-24. https://doi.org/10.1007/PL00006296.

65. Coates BS. Horizontal transfer of a non-autonomous Helitron among insect and viral genomes. BMC Genomics. 2015;16(1):137. https://doi.org/10.1186/ s12864-015-1318-6.

66. Guo X, Gao J, Li F, Wang J. Evidence of horizontal transfer of nonautonomous Lep1 Helitrons facilitated by host-parasite interactions. Sci Rep. 2014;4:5119.

67. Han G, Zhang N, Xu J, Jiang H, Ji C, Zhang Z, et al. Characterization of a novel Helitron family in insect genomes: insights into classification, evolution and horizontal transfer. Mob DNA. 2019;10(1):25. https://doi.org/1 0.1186/s13100-019-0165-4.

68. Tang Z, Zhang HH, Huang K, Zhang XG, Han MJ, Zhang Z. Repeated horizontal transfers of four DNA transposons in invertebrates and bats. Mob DNA. 2015;6(1):3. https://doi.org/10.1186/s13100-014-0033-1.

69. Župunski V, Gubenšek F, Kordis D. Evolutionary dynamics and evolutionary history in the RTE clade of non-LTR retrotransposons. Mol Biol Evol. 2001; 18(10):1849-63. https://doi.org/10.1093/oxfordjournals.molbev.a003727.

70. Chen C, Chen H, Zhang Y, Thomas H, Frank MH, He Y, et al. TBtools: an integrative toolkit developed for interactive analyses of big biological data. Mol Plant. 2020;13(8):1194-202. https://doi.org/10.1016/j.molp.2020.06.009.

71. Gilbert C, Schaack S, Pace JK II, Brindley PJ, Feschotte C. A role for hostparasite interactions in the horizontal transfer of transposons across phyla. Nature. 2010;464(7293):1347-50. https://doi.org/10.1038/nature08939.

72. Zhang HH, Xu HE, Shen $\mathrm{YH}$, Han MJ, Zhang Z. The origin and evolution of six miniature inverted-repeat transposable elements in Bombyx mori and Rhodnius prolixus. Genome Bio Evol. 2013;5(11):2020-31. https://doi.org/10.1 093/gbe/evt153.

73. Lerat $\mathrm{E}$, Rizzon $\mathrm{C}$, Biémont $\mathrm{C}$. Sequence divergence within transposable element families in the Drosophila melanogaster genome. Genome Res. 2003;13(8):1889-96. https://doi.org/10.1101/gr.827603.

74. Chao JT, Kong YZ, Wang Q, Sun YH, Gong DP, LV J, et al. MapGene2Chrom, a tool to draw gene physical map based on Perl and SVG languages. Hereditas. 2015;1:91-7.

75. Lowe TM, Eddy SR. tRNAscan-SE. A program for improved detection of transfer RNA genes in genomic sequence. Nucleic Acids Res. 1997;25(5): 955-64. https://doi.org/10.1093/nar/25.5.955.

76. Edgar RC. MUSCLE: multiple sequence alignment with high accuracy and high throughput. Nucleic Acids Res. 2004;32(5):1792-7. https://doi.org/10.1 093/nar/gkh340.

77. Kumar S, Stecher G, Tamura K. MEGA7: molecular evolutionary genetics analysis version 7.0 for bigger datasets. Mol Biol Evol. 2016;33(7):1870-4 https://doi.org/10.1093/molbev/msw054.

\section{Publisher's Note}

Springer Nature remains neutral with regard to jurisdictional claims in published maps and institutional affiliations.
Ready to submit your research? Choose BMC and benefit from:

- fast, convenient online submission

- thorough peer review by experienced researchers in your field

- rapid publication on acceptance

- support for research data, including large and complex data types

- gold Open Access which fosters wider collaboration and increased citations

- maximum visibility for your research: over $100 \mathrm{M}$ website views per year

At BMC, research is always in progress.

Learn more biomedcentral.com/submissions 